\title{
ON THE QUANTITATIVE QUASI-ISOMETRY PROBLEM: TRANSPORT OF POINCARÉ INEQUALITIES AND DIFFERENT TYPES OF QUASI-ISOMETRIC DISTORTION GROWTH
}

\author{
VLADIMIR SHCHUR
}

\begin{abstract}
We consider a quantitative form of the quasi-isometry problem. We discuss several arguments which lead us to different results and bounds of quasi-isometric distortion: comparison of volumes, connectivity etc. Then we study the transport of Poincaré constants by quasi-isometries and we give sharp lower and upper bounds for the homotopy distortion growth for an interesting class of hyperbolic metric spaces.
\end{abstract}

\section{INTRODUCTION}

In this article we shall study a quantitative form of the quasi-isometry problem: we will give lower and upper bounds for quasi-isometry constants $\lambda$ and $c$ for different classes of spaces. Along the way, we will give a method to transport Poincaré inequalities by quasi-isometries that leads to sharp bounds for certain spaces.

The quantitative quasi-isometry problem consists in evaluating how close two metric spaces can be at various scales, see [8]. Specifically, let $E, F$ be two metric spaces. Consider a ball of radius $R$ in the first space $E$ and take a $(\lambda, c)$-quasi-isometric embedding of this ball in $F$. We are interested in the behaviour of the infimum of the sum $\lambda+c$ of quasi-isometry constants as a function of $R$.

1.1. First examples. Since one may always take $\lambda=1$ and $c=R$, the deviation between any two spaces is at most linear.

Volume considerations show that any space with polynomial volume growth deviates linearly from any space of exponential volume growth (see Proposition 1).

Connectedness considerations provide a lower bound of $\sqrt{R}$ for the embeddings of Euclidean or hyperbolic balls to trees. In the hyperbolic case, this is sharp (see Proposition 3).

This suggests that, in the family of Gromov hyperbolic metric spaces, deviations should be of the order $\sqrt{R}$. Indeed, we show (see Proposition 4) that given two thick enough hyperbolic metric spaces, one can map a $\sqrt{R}$-dense subset of an $R$-ball of the first space into the second one with $\sqrt{R}$ distortion. However, we have been unable to extend such embeddings to the full $R$-ball.

2010 Mathematics Subject Classification. 51F99.

Key words and phrases. Quasi-isometries, hyperbolic spaces, Poincaré constants, quantitative quasiisometry problem. 
There seems to be a rather subtle obstruction to doing this. For instance, we show in Proposition 6 that mapping a tree into hyperbolic space requires linear distortion. This is based on the notion of separation, cf. [1], [2].

1.2. Main result. Our main result is another step towards capturing such obstructions. We shall consider a class of negatively curved locally homogeneous Riemannian manifolds which are not simply connected, but nevertheless hyperbolic. We prove a sharp linear lower bound on the distortion of embeddings which are homotopy equivalences.

Let $\mathbb{T}^{n}$ denote the $n$-dimensional torus. Given positive numbers $\mu_{1} \leq \cdots \leq \mu_{n}$, denote by $Z_{\mu}=\mathbb{T}^{n} \times \mathbb{R}$, where the product space is equipped with the Riemannian metric $d t^{2}+$ $\sum_{i} e^{2 \mu_{i} t} d x_{i}^{2}$. The universal cover of $Z_{\mu}$ is a Riemannian homogeneous space. $Z_{\mu}$ is a hyperbolic metric space. Its ideal boundary is a product of circles, each of which has a metric which is a power of the usual metric. Thus $Z_{\mu}$ can be viewed as a hyperbolic cone over this fractal torus. Essentially our theorem states that the quasi-isometric distortion growth function between such spaces is linear if one requires maps to be isomorphic on fundamental groups.

Theorem 1. (Rough version. For a precise statement, see Theorem [5). Every $(\lambda, c)$ quasi-isometric embedding of an $R$-ball of $Z_{\mu}$ into $Z_{\mu^{\prime}}$ which is a homotopy equivalence satisfies

$$
\lambda+c \geq \operatorname{const}\left(\frac{\sum \mu_{i}}{\mu_{n}}-\frac{\sum \mu_{n}^{\prime}}{\mu_{n}^{\prime}}\right) R .
$$

Conversely, there exist homotopy equivalences with linearly growing distortion,

$$
\lambda+c \leq \text { const } \max \left|\mu_{i}-\mu_{i}^{\prime}\right| R,
$$

from an $R$-ball of $Z_{\mu}$ into $Z_{\mu^{\prime}}$. This is a special case of a more general result which we describe next.

In a hyperbolic metric space, we give a formula for the distance in terms of the visual distance on the ideal boundary. Using this formula we find quasi-isometry constants for the restriction on balls of a map $\Theta$ between $X$ and $Y$ which is a kind of radial extension of a homeomorphism $\theta$ between ideal boundaries. The following is a non technical statement of Theorem 7, see Section 9 for a complete statement.

Theorem 2. Let $X, Y$ be hyperbolic metric spaces. Let $\theta: \partial X \rightarrow \partial Y$ be a homeomorphism. We define the following function. For $R>0$,

$$
K(R)=\sup \left\{\left|\log \frac{d_{y_{0}}\left(\theta\left(\xi_{1}\right), \theta\left(\xi_{2}\right)\right)}{d_{x_{0}}\left(\xi_{1}, \xi_{2}\right)}\right| \mid d_{x_{0}}\left(\xi_{1}, \xi_{2}\right) \geq e^{-R} \vee d_{y_{0}}\left(\theta\left(\xi_{1}\right), \theta\left(\xi_{2}\right)\right) \geq e^{-R}\right\} .
$$

Here $d_{x_{0}}, d_{y_{0}}$ denote visual metrics on ideal boundaries. Then there exists a $(K(R), K(R))$ quasi-isometry between $B_{X}\left(x_{0}, R\right)$ and $B_{Y}\left(y_{0}, R\right)$.

For spaces $Z_{\mu}$, we show that $K(R)=\max _{i}\left|\mu_{i} / \mu_{i}^{\prime}-1\right| R$. Then we give an example of a pair of non-quasi-isometric negatively curved locally homogeneous manifolds and a homeomorphism $\theta$ between their ideal boundaries with $K(R) \lesssim \log R$. This shows that 
subpolynomial (possibly logarithmic) distortion growths also occur in the world of hyperbolic metric spaces.

1.3. Proof. The proof of Theorem 1 involves several results which could have an independent interest and more applications. First, we study the transport of Poincaré inequalities by quasi-isometries. For this purpose we will use kernels to regularize transported functions. Kernels allow us to transport functions from $Y$ to $X$ while controlling quantitatively their Poincaré constants.

Now we give more details on the proof of the theorem itself. It has several steps. First we introduce non-trivial double-covering spaces $\tilde{Z}$ and $\tilde{Z}^{\prime}$ of $Z=Z_{\mu}$ and $Z^{\prime}=Z_{\mu^{\prime}}$. We prove that $\Theta$ lifts to a $\left(\lambda_{1}, 2 c_{1}\right)$-coarse Lipschitz map. Then we take the test-function $e^{\pi i x_{n}}$ on $\tilde{Z}^{\prime}$ which depends only on one coordinate $x_{n}$. It varies very slowly outside of some ball, so the absolute value of the transported and regularised function $v$ on $\tilde{Z}$ stays close to 1 . Lemmas 3 and 4 allow us to control how the lower bound of Poincaré constant changes under transport. This helps us get a lower bound for the Poincaré constant of $\tilde{Z}$ in terms of $\left\{\mu_{i}\right\},\left\{\mu_{i}^{\prime}\right\}$ and the constants of quasi-isometric embedding. We also prove an upper bound for the Poincaré constant of $\tilde{Z}$ in Theorem 3. The combination of these results provides a lower bound for the homotopy distortion growth for $Z$ and $Z^{\prime}$.

\section{BASIC DEFInitions}

Definition 1. Two metric spaces $X$ and $Y$ are said to be roughly quasi-isometric if there exists a pair of maps $f: X \rightarrow Y, g: Y \rightarrow X$ and two constants $\lambda>0$ and $c \geq 0$ such that

- $|f(x)-f(y)| \leq \lambda|x-y|+c$ for every $x, y \in X$,

- $\left|g\left(x^{\prime}\right)-g\left(y^{\prime}\right)\right| \leq \lambda\left|x^{\prime}-y^{\prime}\right|+c$ for every $x^{\prime}, y^{\prime} \in Y$,

- $|g(f(x))-x| \leq c$ for every $x \in X$

- $\left|f\left(g\left(x^{\prime}\right)\right)-x^{\prime}\right| \leq c$ for every $x^{\prime} \in Y$.

The word rough is often dropped away.

The first two conditions mean that $f$ and $g$ are nearly Lipschitz if we are looking from afar. The two latter conditions provide that $f$ and $g$ are nearly inverse of each other. It is easy to check that the composition of two quasi-isometries is also a quasi-isometry. So, quasi-isometries provide an equivalence relation on the class of metric spaces.

Remark 1. Definition 1 is invariant under taking inverse maps.

Definition 2. A map $f: E \rightarrow F$ between metric spaces is a rough $\left(\lambda_{1}, c_{1}, \lambda_{2}, c_{2}\right)$-quasiisometric embedding if for any two points $x, y$ of $E$

$$
\frac{1}{\lambda_{2}}\left(|x-y|_{E}-c_{2}\right) \leq|f(x)-f(y)|_{F} \leq \lambda_{1}|x-y|_{E}+c_{1}
$$

This definition includes quasi-isometries (with $\lambda_{1}=\lambda_{2}$ and $c_{1}=c_{2}$ ) but it does not require the existence of a nearly inverse map. We introduced four constants instead of two because for our quantitative questions we would like to follow what is the role of each inequality in this definition. 
We introduce the following definition to formalize our quantitative problem.

Definition 3. Let $X, Y$ be metric spaces, $x_{0}, y_{0}$ their base points respectively. The quasiisometric distortion growth is the function

$$
\begin{array}{r}
D_{G}\left(X, x_{0}, Y, y_{0}\right)(R)=\inf \left\{d \mid \exists f: B_{X}\left(x_{0}, R\right) \rightarrow Y \text { a }\left(\lambda_{f}, c_{f}\right)\right. \text {-quasi-isometric embedding } \\
\text { such that } \left.f\left(x_{0}\right)=y_{0} \text { and } d=\lambda_{f}+c_{f}\right\} .
\end{array}
$$

We will study the growth of $D_{G}$ as a function of $R$.

\section{General Discussion}

Here we collect elementary arguments which provide lower bounds on quasi-isometry constants.

3.1. Comparison of volumes. First we will show that comparison of volumes in the domain and in the range plays an important role.

By volume of a subset in a metric space, we mean the number of balls of a fixed radius needed to cover that subset.

Consider a space $X$ with an exponential volume growth (for example, hyperbolic plane $\mathbb{H}^{2}$ ) and a space $Y$ with a polynomial volume growth (for example, euclidean space $\mathbb{R}^{n}$ ), then quasi-isometry constants between balls $B_{R}(X)$ and $B_{R}(Y)$ grow linearly in $R: \lambda_{R}+$ $c_{R}=\Omega(R)$.

Proposition 1. Let $X$ be a space with exponential volume growth and $Y$ be a space with polynomial volume growth. Then for any $(\lambda, c)$-quasi-isometric embedding of a ball $B_{X}(R)$ into $Y$ we have $c \geq$ const $\cdot R$.

For the sake of simplicity, in the proof, we will assume that the volume of a ball $B_{X}(R)$ in $X$ is $e^{R}$ and the volume of a ball $B_{Y}(R)$ in $Y$ is $R^{\alpha}$.

Proof. Let $B_{X}(R)$ be a ball in $X, f: B_{X}(R) \rightarrow Y$ be a $(\lambda, c)$-quasi-isometric embedding. Then the diameter of the image $f\left(B_{X}(R)\right)$ is $\leq 2 \lambda R+c$. Consider a maximal set $S$ of points in $B_{X}(R)$ such that pairwise distances between these points are at least $2 c$. We can estimate the cardinality of $S$ as $\#(S) \sim \operatorname{Vol}\left(B_{X}(R) / \operatorname{Vol}\left(B_{X}(2 c)\right)\right.$. For any two points $s_{1}, s_{2} \in S$ the distance between their images is at least $c / \lambda$. Hence, the volume of $f\left(B_{X}(R)\right)$ is at least $\#(S) \times \operatorname{Vol}\left(B_{Y}(c / \lambda)\right)$.

So, on the one hand $\operatorname{Vol}\left(f\left(B_{X}(R)\right)\right) \leq \operatorname{Vol}\left(B_{Y}(2 \lambda R+c)\right)$ and on the other hand $\operatorname{Vol}\left(f\left(B_{X}(R)\right)\right) \geq \operatorname{Vol}\left(B_{Y}(c / \lambda)\right) \operatorname{Vol}\left(B_{X}(R) / \operatorname{Vol}\left(B_{X}(2 c)\right)\right.$. We get

$$
(c / \lambda)^{\alpha} e^{R-2 c} \leq(2 \lambda R+c)^{\alpha} .
$$

For $R$ big enough, this inequality can be satisfied only if exponential term disappears, that is $c=R / 2$.

Remark 2. The same argument yields lower bounds on quasi-isometry constants between balls of the same radius in spaces of different exponential growths. This does not prevent such spaces from being quasi-isometric. For instance, [9] shows that two regular trees of 
degrees at least 4 are always quasi-isometric. The quasi-isometry provided by [9] does not preserve the distance to a fixed point.

3.2. Connectedness. Another property which can detect a difference in the coarse geometry of two spaces is connectedness. For example if we cut a ball from a tree then it will fall into several components, but this does not happen with hyperbolic plane. First, we define coarse connectivity.

Definition 4. A map $f: X \rightarrow Y$ between two metric spaces is called $c$-connected if for any point $x \in X$ and any real number $\delta>0$ there exists $\varepsilon>0$ such that if a point $x^{\prime} \in X$ satisfies $d\left(x, x^{\prime}\right)<\varepsilon$ then $d\left(f(x), f\left(x^{\prime}\right)\right)<c+\delta$,

Definition 5. 1. A metric space $X$ is called $c$-connected if for any two open sets $U, V \subset X$ such that $X=U \cup V$, the intersection of a $c$-neighbourhood of $U$ and $V$ is not empty: $(U+c) \cap V \neq \emptyset$.

2. Equivalently, a metric space $X$ is $c$-connected if for any two points $x, x^{\prime} \in X$ there exists a $c$-connected map $f:[0,1] \rightarrow X$ such that $f(0)=x$ and $f(1)=x^{\prime}$.

First and second definitions are evidently equivalent.

Now we are ready to illustrate our idea. In the following proposition we can take for example hyperbolic plane as the space $X$.

Proposition 2. Let $X$ be a geodesic metric space. We suppose that for any points $x, y$ and any positive real numbers $R$ and $R^{\prime} \leq R / 2$ the set $B_{x}(R) \backslash B_{y}\left(R^{\prime}\right)$ is connected and nonempty. Let $Y$ be a tree, let $f: B_{x}(R) \rightarrow Y$ be a $\left(\lambda_{1}, \lambda_{2}, c_{1}, c_{2}\right)$-quasi-isometric embedding. Then $R \leq 12 \lambda_{2} c_{1}+4 c_{2}$.

Proof. We are going to prove that there exist three points $x_{1}, x_{2}$ and $x$ such that $x_{1}, x_{2} \in$ $B_{x}(R)$ and the distance $d\left(x_{1}, x_{2}\right)$ is at least $R$. Consider a ball of radius $2 R$ centered in $x_{1}$. By hypothesis, the set $B_{x_{1}}(2 R) \backslash B_{x_{1}}(R)$ is non-empty, hence there exists a point $x_{2}$ such that $2 R>d\left(x_{1}, x_{2}\right) \geq R$. The space $X$ is geodesic, hence now we can take the midpoint of $x_{1} x_{2}$ as $x$.

Denote $y_{i}=f\left(x_{i}\right)$ for $i=1,2$.

For any point $y$ of a geodesic $\left(y_{1}, y_{2}\right) \subset Y$ there exists a point $z \in B_{x}(R)$ such that $d(f(z), y) \leq c_{1}$. This follows from the fact that the image of $\left(x_{1}, x_{2}\right)$ is $c_{1}$-connected by the definition of a quasi-isometric embedding and every $c_{1}$-connected path between $y_{1}$ and $y_{2}$ includes the geodesic $\left(y_{1}, y_{2}\right)$ in its $c_{1}$-neighbourhood.

Now consider a chain of points $\left\{\tilde{x}_{i}\right\}$ connecting $x_{1}, x_{2}$ and such that $d\left(\tilde{x}_{i}, \tilde{x}_{i+1}\right)<c_{1} / \lambda_{1}$. Hence, in the image $d\left(f\left(\tilde{x}_{i}\right), f\left(\tilde{x}_{i+1}\right)\right)<2 c_{1}$ and so there exists $i$ such that $d\left(f\left(\tilde{x}_{i}\right), y\right) \leq 2 c_{1}$. Notice that $Y \backslash B_{y}\left(2 c_{1}\right)$ has several $\left(4 c_{1}-2\right)$-connected components and the distance between these components is at least $4 c_{1}$.

Suppose that a point $z$ is rather far from both $x_{1}$ and $x_{2}: d\left(z, x_{i}\right)>4 \lambda_{2} c_{1}+c_{2}, i=$ 1,2. Suppose also that $R>2\left(4 \lambda_{2} c_{1}+c_{2}\right)$ (if not there is nothing to prove). In the set $B_{x}(R) \backslash B_{z}\left(4 \lambda_{2} c_{1}+c_{2}\right)$ we also find a $c_{1} / \lambda_{1}$-chain. Hence, there exists a point $z^{\prime} \notin$ $B_{z}\left(4 \lambda_{2} c_{1}+c_{2}\right)$ of this path such that $d\left(f\left(z^{\prime}\right), y\right) \leq 2 c_{1}$. Hence, $d\left(f(z), f\left(z^{\prime}\right)\right) \leq 4 c_{1}$ and by property of quasi-isometry $d\left(z, z^{\prime}\right) \leq 4 \lambda_{2} c_{1}+c_{2}$, so $z^{\prime} \in B_{z}\left(4 \lambda_{2} c_{1}+c_{2}\right)$. This leads to 
a contradiction with the hypothesis of the proposition. Hence, for any $y \in\left(y_{1}, y_{2}\right)$ there exists $z^{\prime} \in B_{x_{1}}\left(4 \lambda_{2} c_{1}+c_{2}\right) \cup B_{x_{2}}\left(4 \lambda_{2} c_{1}+c_{2}\right)$ such that $d\left(f\left(z^{\prime}\right), y\right) \leq 2 c_{1}$.

Consider two points $y^{\prime}, y^{\prime \prime}$ on the geodesic $\left(y_{1}, y_{2}\right)$ which are close enough to each other (more precisely $\left.d\left(y^{\prime}, y^{\prime \prime}\right) \leq c_{2} / \lambda_{2}\right)$ and such that respective points $z^{\prime}$ and $z^{\prime \prime}$ (which minimise distances to $y^{\prime}$ and $y^{\prime \prime}$, that is $d\left(y^{\prime}, f\left(z^{\prime}\right)\right) \leq 2 c_{1}$ and $\left.d\left(y^{\prime \prime}, f\left(z^{\prime \prime}\right)\right) \leq 2 c_{1}\right)$ lie in different balls $z^{\prime} \in B_{x_{1}}\left(4 \lambda_{2} c_{1}+c_{2}\right)$ and $z^{\prime \prime} \in B_{x_{2}}\left(4 \lambda_{2} c_{1}+c_{2}\right)$. So, on the one hand $d\left(z^{\prime}, z^{\prime \prime}\right) \geq$ $R-8 \lambda_{2} c_{1}-2 c_{2}$ and on the other hand, by triangle inequality $d\left(f\left(z^{\prime}\right), f\left(z^{\prime \prime}\right)\right) \leq c_{2} / \lambda_{2}+4 c_{1}$. Hence $R-8 \lambda_{2} c_{1}-2 c_{2} \leq \lambda_{2}\left(c_{2} / \lambda_{2}+4 c_{1}\right)+c_{2}=4 \lambda_{2} c_{1}+2 c_{2}$. So we get $R \leq 12 \lambda_{2} c_{1}+4 c_{2}$.

Proposition 2 implies that any quasi-isometric embedding of an $R$-ball in hyperbolic plane to a tree has distorsion at least $\sqrt{R}$. We wonder whether this conclusion is sharp.

Here is a partial answer. Let $X$ be a geodesic metric space. Here we will construct an example of a $(\sqrt{R}, \sqrt{R}, \sqrt{R}, \sqrt{R})$-quasi-isometry of a $R$-ball in $X$ to a $\sqrt{R}$-ball in a tree, up to taking a $\sqrt{R}$-dense subset. In this statement the essential point is that we will consider trees of variable degree which will depend on $R$.

Proposition 3. Let $X$ be a geodesic metric space. For any $R>0$ there exists a $\sqrt{R}$-dense subset $S(R) \subset B_{X}(R)$, a tree $T(R)$ and $a(\sqrt{R}, \sqrt{R}, \sqrt{R}, \sqrt{R})$-quasi-isometric embedding $f_{R}: S(R) \rightarrow T(R)$.

Proof. Consider a ball $B_{X}\left(R, z_{0}\right)$ centered at $z_{0}$. We will define a discrete set of points $S(R)$ generation by generation in the following way. The 0 -generation is the origin $z_{0}$. For each $k$ we pick a maximal $\sqrt{R}$-separated subset in the sphere of radius $k \sqrt{R}$. The resulting set $S(R)$ is $\sqrt{R}$-separated. It is also $3 \sqrt{R}$-dense. Indeed, any point in $B((k+1) \sqrt{R})$ is $\sqrt{R}$-close to some point of the sphere of radius $k \sqrt{R}$, in which the $k$-th generation is $2 \sqrt{R}$ dense, by maximality. In particular, every point of the $(k+1)$ th-generation is at distance $\leq 3 \sqrt{R}$ from at least one point of the $k$ th-generation. This provides us with a tree $T(R)$ with vertex set $S(R)$ : we connect each point of the $(k+1)$ th-generation to a closest point of $k$ th-generation (if the choice is not unique we choose the ancestor arbitrarily). Finally we set the lengths of all edges of the constructed tree $T(R)$ equal to 1 . The diameter of $T(R)$ is $\sim \sqrt{R}$.

Now we will sketch the proof that the induced map $f$ is a $(\sqrt{R}, \sqrt{R}, \sqrt{R}, \sqrt{R})$-quasiisometry. The right-hand quasi-isometric inequality $d(f(x), f(y)) \leq O(\sqrt{R}) d(x, y)$ is automatically verified because the diameter of $T(R)$ is $O(\sqrt{R})$. Conversely, given points $x, y \in S(R), z_{0}, f(x)$ and $f(y)$ form a tripod we median point $u$. The distance $d(f(x), f(y))$ is achieved by an arc from $f(x)$ to $u$ followed by an arc from $u$ to $f(y)$ in the tree. The descending arcs from $f(x)$ to $u$ (resp. from $f(y)$ to $u$ ) consist of jumps in $S(R)$ from generation to generation, each of distance at most $3 \sqrt{R}$. Therefore $d(x, y) \leq 3 \sqrt{R} d(f(x), f(y))$.

In the same manner as in the previous proposition we can construct a $(\sqrt{R}, \sqrt{R}, \sqrt{R}, \sqrt{R})$ quasi-isometry between a ball $B_{T}(R)$ of radius $R$ in a regular tree $T$ of degree $d \geq 2$ and a $\sqrt{R}$-dense subset in a ball $B_{\mathbb{H}^{2}}\left(k \ln d, z_{0}\right)$ in $\mathbb{H}^{2}$.

Proposition 4. For any $R>0$, there exist a $\sqrt{R}$-dense subset $S_{R}$ of a ball $B_{\mathbb{H}^{2}}(R)$ in the hyperbolic plane $\mathbb{H}^{2}$ and $a(\sqrt{R}, \sqrt{R}, \sqrt{R}, \sqrt{R})$-quasi-isometry $f_{R}: B_{T}(R) \rightarrow B_{\mathbb{H}^{2}}(R)$. 
Proof. First we will construct the set $S_{R}$ and the quasi-isometry $f_{R}$ and then we will prove that it is indeed a $(\sqrt{R}, \sqrt{R}, \sqrt{R}, \sqrt{R})$-quasi-isometry. Consider $k$ - th generation $G_{k}$ of vertices in $B_{T}$ (that is, points at distance $k$ from the base point), there are $(d+1) d^{k-1}$ points in it. Consider a circle centered in $z_{0}$ of radius $R_{k}$ (its exact value will be calculated soon) and take a subset $S_{k}$ of this circle consisting of $(d+1) d^{k}$ points, such that distance between them is at least $\sqrt{R}_{k}$. So we have the following relation (up to some multiplicative constants) which appears from the consideration of volumes

$$
\operatorname{Vol}\left(\text { ball of radius } \sqrt{R_{k}}\right)(d+1) d^{k}=\operatorname{Vol}\left(\text { circle of radius } R_{k}\right) \text {. }
$$

For big $R_{k}$ we have approximately

$$
e^{\sqrt{R_{k}}}(d+1) d^{k}=e^{R_{k}} .
$$

We set $R_{0}=0$. Then it follows that $R_{k} \approx k \ln d$. We send points from $G_{k}$ to $S_{k}$ naturally. Now we need to add edges between points of successive sets $S_{k}$. We connect points of $S_{k}$ to the nearest points from $S_{k-1}$. If there are two possibilities, we choose one arbitrary.

Let us show that this is a $(\sqrt{R}, \sqrt{R}, \sqrt{R}, \sqrt{R})$-quasi-isometry. First of all, for any two

points $t_{1}, t_{2} \in S$, the distance between their images is at least $\sqrt{R}$. We have always $d\left(t_{1}, t_{2}\right) \leq R \leq \sqrt{R} d\left(f_{R}\left(t_{1}\right), f_{R}\left(t_{2}\right)\right)+\sqrt{R}$ and this inequality is checked automatically. Now, let $u_{0}=t_{1}, u_{1}, \ldots, u_{n-1}, u_{n}=t_{2}$ be a geodesic path between $t_{1}$ and $t_{2}$. We notice that $d\left(u_{i}, u_{i+1}\right)=1 \geq d\left(f\left(u_{i}\right), f\left(u_{i+1}\right)\right) / \sqrt{R}$ for $i=0,1, \ldots, n-1$. Then

$$
\begin{aligned}
d\left(t_{1}, t_{2}\right)=\sum_{i=0}^{n-1} d\left(t_{i}, t_{i+1}\right) & \geq \sum_{i=0}^{n-1} d\left(f\left(u_{i}\right), f\left(u_{i+1}\right)\right) / \sqrt{R} \geq \\
d\left(f\left(t_{1}\right), f\left(t_{2}\right)\right) / \sqrt{R} & \geq\left(d\left(f\left(t_{1}\right), f\left(t_{2}\right)\right)-\sqrt{R}\right) / \sqrt{R}
\end{aligned}
$$

what finishes the proof.

Though we do not know if we can extend this quasi-isometry to the whole ball $B_{\mathbb{H}^{2}}(R)$. The first idea is to do a projection of $B_{\mathbb{H}^{2}}(R)$ on a discrete subset, but this projection is a $(1,1, \sqrt{R}, \sqrt{R})$-quasi-isometry itself, hence the resulting map is a $(R, R, R, R)$-quasiisometry.

\section{Poincaré inequalities and Quasi-isometries}

4.1. The critical exponent $p_{\neq 0}$ for $L^{p}$-cohomology $L^{p}$-cohomology groups provides invariants for quasi-isometries. The continuous first $L^{p}$-cohomology group of a hyperbolic metric space $X$ is

$$
L^{p} H_{\text {cont }}^{1}(X):=\left\{[f] \in L^{p} H^{1}(X) \mid f \text { extends continuously to } X \cup \partial X\right\},
$$

where $X \cup \partial X$ is Gromov's compactification of $X$. Following the works of Pierre Pansu, and Marc Bourdon and Bruce Kleiner [10], we define the following quasi-isometrical numerical invariant of $X$

$$
p_{\neq 0}(X)=\inf \left\{p \geq 1 \mid L^{p} H_{\text {cont }}^{1}(X) \neq 0\right\} .
$$


If $p_{\neq 0}$ achieves different values for two spaces $X$ and $Y$, then $X$ and $Y$ are not quasiisometric. We expect that the difference $\left|p_{\neq 0}(X)-p_{\neq 0}(Y)\right|$ also bounds from below the quasi-isometrical distortion growth. We are able to prove this only for a family of examples, and under certain restrictions on maps.

Let $Z_{\mu}$ and $Z_{\mu^{\prime}}$ be two variants of the space $\mathbb{T}^{n} \times(-\infty, \infty)$ with metrics $d t^{2}+\sum e^{2 \mu_{i} t} d x_{i}^{2}$ and $d t^{2}+\sum e^{2 \mu_{i}^{\prime} t} d x_{i}^{2}$ respectively. The main result of this part is a sharp lower bound for the quasi-isometrical distortion growth between $Z_{\mu}$ and $Z_{\mu^{\prime}}$, of the form

$$
\text { const }\left(p_{\neq 0}\left(Z_{\mu^{\prime}}\right)-p_{\neq 0}\left(Z_{\mu}\right)\right) R \text {. }
$$

4.2. Definition of Poincaré constants. Constants in Poincaré inequalities are the quantitative incarnation of $L^{p}$-cohomology. On Riemannian manifolds, Poincaré inequality is defined as follows.

Definition 6. Let $X$ be a Riemannian manifold. We say that $X$ satisfies Poincaré inequality if there exists a real number $C$ such that for any real valued function $f$ on $X$, there exists a real number $m_{f}$ such that

$$
\left\|f-m_{f}\right\|_{p} \leq C\|\nabla f\|_{p} .
$$

The best constant $C$, denoted by $C_{p}(X)$, is called Poincaré constant of $X$.

We are not satisfied by this definition as we want to work with a wider class of metric spaces. The generalization involves semi-norms induced by kernels (see Definitions 7, 9). Let $\psi$ be a kernel on $X$. The semi-norm $N_{p, \psi}(f)$ is an analog of the $\mathbb{L}^{p}$-norm of the gradient on a Riemannian manifold.

First we recall what are kernels on geodesic metric spaces.

Definition 7. Let $X$ be a geodesic space, $d x$ a Radon measure on $X$. A kernel $\psi$ is a measurable non-negative function on $X \times X$ such that

- $\psi$ is bounded, $\psi \leq S^{\psi}$;

- for every $x \in X \int_{X} \psi\left(x, x^{\prime}\right) d x^{\prime}=1$;

- the support of $\psi$ is concentrated near the diagonal: there exist constants $\varepsilon^{\psi}>0$, $\tau^{\psi}>0$ and $R^{\psi}<\infty$ such that $\psi(x, y)>\tau^{\psi}$ if $d(x, y) \leq \varepsilon^{\psi} ; \psi(x, y)=0$ if $d(x, y)>R^{\psi}$.

$R^{\psi}$ is called the width, $\varepsilon^{\psi}$ - the radius of positivity, $S^{\psi}$ - the supremum and $\tau^{\psi}$ - the margin of $\psi$.

Definition 8. A cocycle on $Y$ is a measurable map $a: Y \times Y \rightarrow \mathbb{R}$ such that for every $y_{1}, y_{2}, y_{3}$ in $Y$,

$$
a\left(y_{1}, y_{2}\right)=a\left(y_{1}, y_{3}\right)+a\left(y_{2}, y_{3}\right) .
$$

The convolution of a cocycle with a kernel is defined by

$$
a * \phi\left(x, x^{\prime}\right)=\int_{Y \times Y} a\left(y, y^{\prime}\right) \phi(x, y) \phi\left(x^{\prime}, y^{\prime}\right) d y d y^{\prime} .
$$


Definition 9. Let $\psi$ be a kernel and $a$ a cocycle on $X$. The semi-norm $N_{p, \psi}$ is defined by

$$
N_{p, \psi}(a)=\left(\int_{X \times X}\left|a\left(x_{1}, x_{2}\right)\right|^{p} \psi\left(x_{1}, x_{2}\right) d x_{1} d x_{2}\right)^{1 / p} .
$$

For $f$ a measurable function on $X$,

$$
N_{p, \psi}(f)=\left(\int_{X \times X}\left|f\left(x_{1}\right)-f\left(x_{2}\right)\right|^{p} \psi\left(x_{1}, x_{2}\right) d x_{1} d x_{2}\right)^{1 / p} .
$$

Definition 10. The Poincaré inequality associated with a kernel $\psi$ is

$$
\left\|f-m_{f}\right\|_{p} \leq C_{p}(X, \psi) N_{p, \psi}(f) .
$$

4.3. Scheme of proof of a lower bound on distorsion. For the family of spaces $Z_{\mu}$, it is known that $p_{\neq 0}\left(Z_{\mu}\right)=\frac{\sum \mu_{i}}{\max \mu_{i}}$ (unpublished result of P. Pansu). In Theorem 5 we show that

- if $p>p_{\neq 0}\left(Z_{\mu}\right)$, then the Poincaré constant for a ball of radius $R$ satisfies

$$
C_{p}\left(B^{Z_{\mu}}(R)\right) \geq \text { const. }(\operatorname{Vol} B(R))^{1 / p}
$$

- if $p \leq p_{\neq 0}\left(Z_{\mu}\right)$, then

$$
C_{p}\left(B^{Z_{\mu}}(R)\right)=o\left((\operatorname{Vol} B(R))^{1 / p}\right) .
$$

Next, we show that under transport by a $(\lambda, c)$-quasi-isometry, $C_{p}$ is multiplied by at most $e^{(\lambda+c) / a}$ for some positive constant $a$. Transport under quasi-isometric embeddings is more delicate, this is why our arguments work only for a family of examples. For these examples, we are able to get a lower bound. Roughly speaking, it states

Assume that $p_{\neq 0}\left(Z_{\mu^{\prime}}\right)<p<p_{\neq 0}\left(Z_{\mu}\right)$. If there exists a $(\lambda, c)$-quasi-isometric embedding $B^{Z_{\mu}}(R) \rightarrow Z_{\mu^{\prime}}$, which induces an isomorphism on fundamental groups, then

$$
C_{p}\left(B^{Z_{\mu}}(R)\right) \geq \text { const. } e^{-(\lambda+c) / a} C_{p}\left(B^{Z_{\mu^{\prime}}}(R)\right) .
$$

This yields

$$
\begin{aligned}
\lambda+c & \geq a\left(\log \left(C_{p}\left(B^{Z_{\mu^{\prime}}}(R)\right)\right)-\log \left(C_{p}\left(B^{Z_{\mu}}(R)\right)\right)\right. \\
& \sim\left(p_{\neq 0}\left(Z_{\mu^{\prime}}\right)-p_{\neq 0}\left(Z_{\mu}\right)\right) R .
\end{aligned}
$$

which is the announced lower bound on quasi-isometric distortion growth.

\section{Regularisation AND QUASI-ISOMETRIES}

In this section we will study how Poincaré inequalities are transformed under quasiisometries. For this purpose we will use kernels, which will help us to regularize transported functions. 
5.1. Kernels. The convolution of two kernels is

$$
\psi_{1} * \psi_{2}=\int_{X} \psi_{1}(x, z) \psi_{2}(z, y) d z
$$

the result is also a kernel. The convolution of a kernel and a function is

$$
g * \psi(x)=\int_{X} g(z) \psi(x, z) d z .
$$

Lemma 1. There exists a constant $c_{\tau}$ (which depends on the local geometry of the space $X)$ such that for any $\varepsilon>0$ there exists $\tau=c_{\tau} e^{-\varepsilon}$ and a kernel $\psi$ on $X \times X$ such that for any two points $x_{1}, x_{2}$ with $d\left(x_{1}, x_{2}\right)<\varepsilon$, we have $\psi\left(x_{1}, x_{2}\right)>\tau$. In other words, for any given radius of positivity $\varepsilon$ there exists a kernel with a margin controlled from below by $c_{\tau} e^{-\varepsilon}$.

Proof. We start from kernel

$$
\psi^{\prime}\left(x, x^{\prime}\right)=\operatorname{Vol}(B(x, 1))^{-1} 1_{\left\{d\left(x, x^{\prime}\right) \leq 1\right\}}
$$

with radius of positivity $\varepsilon^{\prime}=1$ and margin $\tau^{\prime}=v(1)^{-1}$, where, for $r>0, v(r)$ denotes the infimum of volumes of balls of radius $r$ in $X$. We know from the proof of Lemma 1.2 in [3] that the $m$-th convolution $\psi^{\prime * m}$ has radius of positivity $\varepsilon_{m}^{\prime} \geq m\left(\varepsilon^{\prime} / 2\right)=m / 2$ and margin $\tau_{m}^{\prime} \geq \tau^{\prime m} v\left(\frac{1}{2}\right)^{m-1}$. We denote $v\left(\frac{1}{2}\right)^{m-1}$ by $c_{\tau}$ which finishes the proof.

The following facts are known, see [3].

Lemma 2. Let $X$ be a geodesic metric space such that the infimum $\inf \{\operatorname{Vol} B(x, r) \mid x \in X\}$ of volume of balls of radius $r$ is positive. Semi-norms $N_{p, \psi}$ are pairwise equivalent. More precisely, let $\psi_{1}$ and $\psi_{2}$ be two kernels on $X$. Then

$$
N_{\psi_{2}} \leq \hat{C} N_{\psi_{1}}
$$

where

$$
\hat{C}=\frac{\sup \psi_{1} \sup \psi_{2}}{c_{\tau}} \frac{R^{\psi_{2}}}{\varepsilon^{\psi_{1}}}(2 e)^{R^{\psi_{2}} / \varepsilon^{\psi_{1}}}
$$

Lemma 3. Let the space $X$ be a Riemannian manifold and have the following properties: (1) its injectivity radius is bounded below, (2) its Ricci curvature is bounded from below. Then the volumes of balls are bounded from below (Croke inequality [11]) and from above (Bishop inequality).

1) For any function $g$ define a cocycle $u(x, y)=g(x)-g(y)$. Then for any $p$ and any kernel $\psi^{\prime}$ with bounded derivatives there exists a kernel $\psi_{1}$ such that the $\mathbb{L}^{p}$-norm of $\nabla\left(g * \psi^{\prime}\right)$ (we regularise $g$ ) is bounded from above by a $\psi_{1}$-seminorm of the corresponding cocycle $u$

$$
\left\|\nabla\left(g * \psi^{\prime}\right)\right\|_{p} \leq N_{p, \psi_{1}}(u)
$$

with the kernel $\psi_{1}$ defined as follows

$$
\psi_{1}=\frac{\sup \nabla \psi^{\prime} \sup \psi^{\prime}}{\operatorname{Vol}\left(B\left(z^{\prime}, R^{\psi^{\prime}}\right)\right)} 1_{\left\{d\left(z, z^{\prime}\right) \leq R^{\psi^{\prime}}\right\}} .
$$


2) Conversely, there also exists a kernel $\psi_{2}$ such that

$$
N_{p, \psi_{2}}(u) \leq C\|\nabla g\|_{p},
$$

where $C$ depends only on dimension. Here the kernel $\psi_{2}$ can be taken as

$$
\psi_{2}(x, y)=\max \left\{1, \Theta(x, y)^{-1}\right\} 1_{\{d(x, y) \leq R\}},
$$

where $\Theta(x, y)$ is the density of the volume element in polar coordinates with origin at $x$

$$
\Theta(x, y)^{-1} d y=d r d \theta
$$

and $R>0$ can be chosen arbitrarily.

In the third hypothesis we propose to use $R=1$, then $\psi_{2}$ is bounded by 1 and the width of its support is also 1. For reader's convenience, we include the proof of the first statement of the last Lemma, following [3].

Proof. Denote by $\alpha$ the cocycle $u * \psi^{\prime}$. Then for any $y$,

$$
\nabla\left(u * \psi^{\prime}\right)(x)=\frac{\partial \alpha(x, y)}{\partial x}=\int\left(g\left(z^{\prime}\right)-g(z)\right) d_{x} \psi^{\prime}(z, x) \psi^{\prime}\left(z^{\prime}, y\right) d z d z^{\prime} .
$$

Choose $y=x$. Then we obtain

$$
\left|\nabla\left(g * \psi^{\prime}(x)\right)\right| \leq \sup \nabla \psi^{\prime} \sup \psi \int_{B\left(x, R^{\psi}\right) \times B\left(x, R^{\psi}\right)}\left|g\left(z^{\prime}\right)-g(z)\right| d z d z^{\prime} .
$$

Now applying Hölder inequality we get the needed statement with the kernel

$$
\psi_{1}=\frac{\sup \nabla \psi^{\prime} \sup \psi^{\prime}}{\operatorname{Vol}\left(B\left(z^{\prime}, R^{\psi^{\prime}}\right)\right)} 1_{\left\{d\left(z, z^{\prime}\right) \leq R^{\psi^{\prime}}\right\}} .
$$

This lemma gives us an idea how to generalize Poincaré inequalities for the case of arbitrary metric spaces. Of course, such Poincaré inequality depends on a choice of a kernel $\psi$. Let $f$ be an $\mathbb{L}_{p}$-function on $X, \psi$ a kernel on $X$. The Poincaré inequalities for $f$ associated to $\psi$ with constants $c_{f}$ and $C_{p}(f)$ is

$$
\left\|f-c_{f}\right\|_{p} \leq C_{p}(f)\left\|N_{p, \psi}(u)\right\| .
$$

The Poincaré constant $C_{p}(X, \psi)$ is a constant such that for any $\mathbb{L}_{p}$-function $f$ Poincaré inequality is checked with $C_{p}(f)=C_{p}(X, \psi)$. It follows from Lemma 2 that the existence of Poincaré constant does not depend on the choice of a kernel.

5.2. Transporting functions by quasi-isometries. Let $X, Y$ be two metric spaces, let $f: X \rightarrow Y$ and $f^{\prime}: Y \rightarrow X$ be $(K, c)$-quasi-isometries between them such that for any $x \in X, d\left(x, f^{\prime} \circ f(x)\right) \leq c$ and vice versa (that is, they are inverse in the quasi-isometrical sense). Let $g$ be a measurable function on $Y$. We want to find a way to transport and to regularize $g$ by our quasi-isometry to obtain a similar measurable function on $X$. We will take

$$
h(x)=\int_{Y} g(z) \psi(f(x), z) d z
$$


as a function on $X$ corresponding to $g$. This integral exists for all $x$ because $\psi$ is measurable by the second variable by definition. Still we want $h$ to be also measurable. For that, it will be sufficient if $f$ is measurable too.

Proposition 5. Let $f$ be a $\left(\lambda_{1}, \lambda_{2}, c_{1}, c_{2}\right)$-quasi-isometric embedding between metric spaces $X$ and $Y$. Then there exists a measurable $\left(\lambda_{1}, \lambda_{2}, 3 c_{1}, c_{2}+2 c_{1} / \lambda_{1}\right)$-quasi-isometric embedding $g$ at distance $2 c_{1}$ from $f$.

Proof. Take a measurable partition $P$ of $X$ with a mesh $c_{1} / \lambda_{1}$. For each set $A \in P$ we choose a base point $x_{A}$. We set $g$ be constant on $A$

$$
\left.g\right|_{A}=f\left(x_{A}\right) .
$$

Take any two points $x, x^{\prime} \in X$. Assume $x \in A$ and $x^{\prime} \in A^{\prime}$ where $A, A^{\prime} \in P$. Then

$$
\begin{aligned}
d\left(g(x), g\left(x^{\prime}\right)\right) & =d\left(f\left(x_{A}\right), f\left(x_{A^{\prime}}\right)\right) \leq \lambda_{1} d\left(x_{A}, x_{A}^{\prime}\right)+c_{1} \\
& \leq \lambda_{1}\left(d\left(x, x^{\prime}\right)+d\left(x, x_{A}\right)+d\left(x^{\prime}, x_{A^{\prime}}\right)\right)+c_{1} \leq \lambda_{1} d\left(x, x^{\prime}\right)+3 c_{1} .
\end{aligned}
$$

In the same way we prove the right-hand inequality.

This proposition gives us an idea that we can always pass to measurable quasi-isometries without significant loss in constants. From now we will consider only measurable quasiisometries.

\subsection{Transporting cocycles.}

Definition 11. Let $a$ be a cocycle on $Y, f: X \rightarrow Y$ be a quasi-isometric embedding and $\phi$ be a kernel on $Y$. The transporting convolution of $a$ with $\phi$ by $f$ is the cocycle defined on $X$ by

$$
a *_{t} \phi(f)\left(x, x^{\prime}\right)=\int_{Y \times Y} a\left(y, y^{\prime}\right) \phi(f(x), y) \phi\left(f\left(x^{\prime}\right), y^{\prime}\right) d y d y^{\prime} .
$$

Lemma 4. Let $X, Y$ be two metric space. Suppose also that $X$ has a bounded geometry (that is for any $R>0$ the supremum of volume of balls of radius $R$ in $X$ is bounded). Let $\phi$ be a kernel on $Y$, let a be a cocycle on $Y$ and let $\psi$ be a kernel on $X$. Let also $f$ be $a$ $\left(\lambda_{1}, \lambda_{2}, c_{1}, c_{2}\right)$-quasi-isometric embedding. Then there exists a kernel $\tilde{\psi}$ on $Y$ such that

$$
N_{\psi}\left(a *_{t} \phi(f)\right) \leq C N_{\tilde{\psi}}(a)
$$

where

$$
C \leq\left(c_{\tau}^{Y}\right)^{-1} e^{R^{\psi^{\prime}}} \sup \psi\left(\sup \phi \sup \operatorname{Vol} B_{X}\left(2 \lambda_{2} R^{\phi}+c_{2}\right)\right)^{2} .
$$

Proof. By definition,

$$
\begin{array}{r}
\left(N_{\psi}\left(a *_{t} \phi(f)\right)\right)^{p}=\int_{X \times X}\left|a *_{t} \phi\left(x, x^{\prime}\right)\right|^{p} \psi\left(x, x^{\prime}\right) d x d x^{\prime}= \\
=\int_{X \times X}\left|\int_{Y \times Y} a\left(y, y^{\prime}\right) \phi(f(x), y) \phi\left(f\left(x^{\prime}\right), y^{\prime}\right) d y d y^{\prime}\right|^{p} \psi\left(x, x^{\prime}\right) d x d x^{\prime}
\end{array}
$$


applying Hölder inequality

$$
\leq \int_{X \times X} \int_{Y \times Y}\left|a\left(y, y^{\prime}\right)^{p}\right| \phi(f(x), y) \phi\left(f\left(x^{\prime}\right), y^{\prime}\right) d y d y^{\prime} \psi\left(x, x^{\prime}\right) d x d x^{\prime}
$$

denoting $\psi^{\prime}\left(y, y^{\prime}\right)=\int_{X \times X} \phi(f(x), y) \phi\left(f\left(x^{\prime}\right), y^{\prime}\right) \psi\left(x, x^{\prime}\right) d x d x^{\prime}$

$$
=\int_{Y \times Y}\left|a\left(y, y^{\prime}\right)\right|^{p} \psi^{\prime}\left(y, y^{\prime}\right) d y d y^{\prime} .
$$

We need to show that $\psi^{\prime}$ is dominated by some kernel $\tilde{\psi}$.

First we will prove that $\psi^{\prime}\left(y, y^{\prime}\right)=0$ if $d\left(y, y^{\prime}\right)>R^{\psi^{\prime}}$ for some $R^{\psi^{\prime}}=R^{\phi}+\lambda R^{\psi^{\prime}}+c$. If $d\left(x, x^{\prime}\right)>R^{\psi}$ then by the definition of kernels $\psi\left(x, x^{\prime}\right)=0$. Otherwise, suppose that $d\left(x, x^{\prime}\right)<R^{\psi^{\prime}}$. If $d\left(y, y^{\prime}\right)>R^{\psi^{\prime}}$, then by triangle inequality either $\phi(f(x), y)$ or $\phi\left(f\left(x^{\prime}\right), y^{\prime}\right)$ vanishes:

$$
d\left(f(x), f\left(x^{\prime}\right)\right) \leq \lambda d\left(x, x^{\prime}\right)+c \leq \lambda R^{\psi^{\prime}}+c .
$$

Hence, if, for example, $d(f(x), y) \leq R^{\phi}$, then $d\left(f\left(x^{\prime}\right), y^{\prime}\right) \geq R^{\prime}-d\left(f(x), f\left(x^{\prime}\right)\right)>R^{\phi}$ which leads to $\phi\left(f\left(x^{\prime}\right), y^{\prime}\right)=0$.

We estimate $\psi^{\prime}\left(y, y^{\prime}\right)$ from above in the following way. First we write

$$
\psi^{\prime}\left(y, y^{\prime}\right) \leq \sup \psi \int_{X \times X} \phi(f(x), y) \phi\left(f\left(x^{\prime}\right), y^{\prime}\right) d x d x^{\prime} .
$$

Now we have to integrate $\int_{X} \phi(f(x), y) d x$ and $\int_{X} \phi\left(f\left(x^{\prime}\right), y^{\prime}\right) d x^{\prime}$.

For any $y \in Y$, if $d(f(x), y)>R^{\phi}$ then $\phi(f(x), y)=0$. Hence, the diameter of the set of points $X_{y} \in X$ such that for any $x \in X_{y} d(f(x), y) \leq R^{\phi}$, is at most $\lambda_{2} 2 R^{\phi}+c_{2}$. Hence, $\int_{X} \phi(f(x), y) d x \leq\left(\sup _{x \in X} \operatorname{Vol} B_{X}\left(x, 2 \lambda_{2} R^{\phi}+c_{2}\right)\right) \sup _{Y \times Y} \phi$, that is $\sup _{x \in X} \operatorname{Vol} B_{X}\left(x, 2 \lambda_{2} R^{\phi}+\right.$ $c_{2}$ ) stands for the supremum of volumes of all balls of radius $2 \lambda_{2} R^{\psi}+c_{2}$ in $X$. So we come to the following upper-bound for $\psi^{\prime}\left(y, y^{\prime}\right)$

$$
\psi^{\prime}\left(y, y^{\prime}\right) \leq \sup \psi\left(\sup \phi \sup \operatorname{Vol} B_{X}\left(2 \lambda_{2} R^{\phi}+c_{2}\right)\right)^{2}
$$

Lemma 1 helps us to construct a kernel $\tilde{\psi}$ such that its radius of positivity is at least $R^{\psi^{\prime}}$ and at the same time we control its margin from below. $\tilde{\psi}\left(y, y^{\prime}\right) \geq \tau=c_{\tau}^{Y} e^{-R^{\psi^{\prime}}}$ whenever the distance between $y, y^{\prime}$ does not exceed $R^{\psi^{\prime}}$. Hence,

$$
\psi^{\prime}\left(y, y^{\prime}\right) \leq \tau^{-1} \tilde{\psi}\left(y, y^{\prime}\right) \sup \psi\left(\sup \phi \sup \operatorname{Vol} B_{X}\left(2 \lambda_{2} R^{\phi}+c_{2}\right)\right)^{2} .
$$

So, we obtain

$$
C \leq\left(c_{\tau}^{Y}\right)^{-1} e^{R^{\psi^{\prime}}} \sup \psi\left(\sup \phi \sup \operatorname{Vol} B_{X}\left(2 \lambda_{2} R^{\phi}+c_{2}\right)\right)^{2}
$$




\section{Poincare inequality for exponential metric}

We will give an upper bound for the Poincaré constant in a ball of radius $R$ in a space with the metric $d t^{2}+\sum_{i} e^{2 \mu_{i} t} d x_{i}^{2}$.

Theorem 3. Let $\tilde{X}=\mathbb{R}_{+} \times \mathbb{R}^{n}$ with the metric $d t^{2}+\sum_{i} e^{2 \mu_{i} t} d x_{i}^{2}$. Let $X=\tilde{X} / \Gamma$ where $\Gamma$ is a lattice of translations in the factor $\mathbb{R}^{n}$. Then the Poincaré constant for a ball $B(R)$ in $X$ is

$$
C_{p}(\mu) \leq \frac{p}{\mu}+(A(\mu))^{1 / p} C_{p}\left(\mathbb{T}^{n}\right) e^{\mu_{n} R}
$$

where $\mu=\sum \mu_{i}, A(\mu)$ is a constant depending only on $\mu, C_{p}\left(\mathbb{T}^{n}\right)$ is a Poincaré constant for a torus $\mathbb{T}^{n}$.

First, we fix the direction $\theta=\left(x_{1}, \ldots, x_{n}\right)$.

\subsection{Poincaré inequality in a fixed direction.}

Lemma 5. Let $\tilde{X}=\mathbb{R}_{+} \times \mathbb{R}^{n}$ with the metric $d t^{2}+\sum_{i} e^{2 \mu_{i} t} d x_{i}^{2}$. Let $X=\tilde{X} / \Gamma$ where $\Gamma$ is a lattice of translations in the factor $\mathbb{R}^{n}$. Let $R \in \mathbb{R}^{+} \cup\{\infty\}$. Then for any fixed direction $\theta=\left(x_{1}, \ldots, x_{n}\right)$

$$
\left(\int_{a}^{R}\left|f(t)-c_{\theta}\right|^{p} e^{\mu t} d t\right)^{1 / p} \leq \frac{p}{\mu}\left(\int_{a}^{R}\left|f^{\prime}(t)\right|^{p} e^{\mu t} d t\right)^{1 / p}
$$

where $c_{\theta}=f(R, \theta)$ or $c_{\theta}=\lim _{R \rightarrow \infty} f(R, \theta)$.

Proof. Let $f$ be a function such that its partial derivative $\partial f / \partial t$ is in $\mathbb{L}^{p}\left(e^{\mu t} d t,[0,+\infty)\right)$ where $p>1$. By Hölder inequality we get

$$
\int_{0}^{+\infty}\left|\frac{\partial f}{\partial t}\right| d t \leq\left(\int_{0}^{+\infty}\left|\frac{\partial f}{\partial t}\right|^{p} e^{\mu t} d t\right)^{1 / p}\left(\int_{0}^{+\infty} e^{-(\mu t / p)(p /(p-1))}\right)^{1-1 / p}<+\infty .
$$

Hence, for every fixed direction $\theta$ there exists a $\operatorname{limit}_{t \rightarrow \infty} f(t, \theta)$.

First, if $R=\infty$, we prove that $\left|f(t)-c_{\theta}\right|^{p} e^{\mu t} \rightarrow 0$ as $t \rightarrow \infty$. We apply the NewtonLeibniz theorem and then Hölder inequality to $\left|f(t)-c_{\theta}\right|$. We have

$$
\begin{array}{r}
\left|f(t)-c_{\theta}\right|=\left|\int_{t}^{\infty} \frac{\partial f}{\partial s} d s\right| \leq \int_{t}^{\infty}\left|\frac{\partial f}{\partial s}\right| d s \leq \\
\leq\left(\int_{t}^{\infty}\left|\frac{\partial f}{\partial s}\right|^{p} e^{\mu u} d u\right)^{1 / p}\left(\int_{t}^{\infty} e^{-\mu s /(p-1)} d s\right)^{1-1 / p} .
\end{array}
$$

We calculate the last integral

$$
\int_{t}^{\infty} e^{-\mu s /(p-1)} d s=-\left.\frac{p-1}{\mu} e^{-\frac{\mu s}{p-1}}\right|_{t} ^{\infty}=\frac{p-1}{\mu} e^{-\frac{\mu t}{p-1}} .
$$

With the notation $D_{0}=\left(\frac{p-1}{\mu}\right)^{p-1}$,

$$
\left|f(t)-c_{\theta}\right|^{p} \leq D_{0} e^{-\mu t} \int_{t}^{+\infty}\left|\frac{\partial f}{\partial s}\right|^{p} e^{\mu s} d s .
$$


Hence

as $t \rightarrow+\infty$.

$$
\left|f(t)-c_{\theta}\right|^{p} e^{\mu t} \leq D_{0} \int_{t}^{+\infty}\left|\frac{\partial f}{\partial s}\right|^{p} e^{\mu s} d s \rightarrow 0
$$

Now we integrate by parts

$$
\int_{a}^{R}\left|f(t)-c_{\theta}\right|^{p} e^{\mu t} d t=\left[\left|f(t)-c_{\theta}\right|^{p} \frac{e^{\mu t}}{\mu}\right]_{a}^{R}-\int_{a}^{R} f^{\prime}(t) p\left|f(t)-c_{\theta}\right|^{p-1} \frac{e^{\mu t}}{\mu} d t .
$$

As $c_{\theta}=f(R)$

$$
\int_{a}^{R}\left|f(t)-c_{\theta}\right|^{p} e^{\mu t} d t=-\left|f(a)-c_{\theta}\right|^{p} \frac{e^{\mu a}}{\mu}-p \int_{a}^{R} f^{\prime}(t)\left|f(t)-c_{\theta}\right|^{p-1} \frac{e^{\mu t}}{\mu} d t .
$$

We notice that the integral at the left is positive. On the right hand side, the first term is negative (for this reason we will drop it soon). Hence, the second term should be positive. By Hölder inequality,

$$
\int_{a}^{R}\left(-f^{\prime}(t)\right)\left|f(t)-c_{\theta}\right|^{p-1} \frac{e^{\mu t}}{\mu} d t \leq\left(\int_{a}^{R}\left|f^{\prime}(t)\right|^{p^{e^{\mu t}}} \frac{1}{\mu} d t\right)^{1 / p}\left(\int_{a}^{R}\left|f(t)-c_{\theta}\right|^{p} \frac{e^{\mu t}}{\mu} d t\right)^{(p-1) / p} .
$$

We introduce the following notations

$$
X=\int_{a}^{R}\left|f(t)-c_{\theta}\right|^{p} e^{\mu t} d t, \quad Y=\int_{a}^{R}\left|f^{\prime}(t)\right|^{p} e^{\mu t} d t
$$

Using these notations we return to Eq. (2). First we drop the term $-\left|f(a)-c_{\theta}\right|^{p} e^{\mu a} / \mu$ and then we apply Eq. (3)

$$
X \leq \frac{p}{\mu} Y^{1 / p} X^{(p-1) / p}
$$

So, we get immediately that

$$
X^{1 / p} \leq \frac{p}{\mu} Y^{1 / p}
$$

which proves Poincaré inequality in a fixed direction.

6.2. Poincaré inequality for exponential metric. Here we will finish the proof of Theorem 3. We introduce the following notations $\tilde{f}_{r}(t, \theta)=f(r, \theta)$ (the function is considered as a function of two variables), $f_{r}(\theta)=f(r, \theta)$ (the function is considered as a function of one variable).

We have already proved that for any $\theta \in \mathbb{T}^{n}$,

$$
\int_{0}^{R}|f(t, \theta)-f(R, \theta)|^{p} e^{\mu t} d t \leq\left(\frac{p}{\mu}\right)^{p} \int_{0}^{R}\left|\frac{\partial f}{\partial t}\right|^{p} e^{\mu t} d t .
$$

We integrate over $\theta$ and we introduce the volume element for $\tilde{X}, d V o l=d r d \theta e^{\sum \mu_{i} r}$. We get

$$
\int_{B(R)}\left|f-f_{R}\right|^{p} d V o l \leq\left(\frac{p}{\mu}\right)^{p} \int_{B(R)}|\nabla f|^{p} d V o l .
$$


Denote the Euclidean gradient by $\nabla_{e}$. By the form of the metric we see that $e^{2 \mu_{i} t}\left|d x_{i}^{2}\right|=$ 1. Hence, $\left\|\nabla_{e} f_{r}\right\| \leq e^{\mu_{n} t}|\nabla f|$. Now we notice that

$$
\int_{R-1}^{R}\left\|\nabla_{e} f_{r}\right\|_{\mathbb{L}^{p}\left(\mathbb{T}^{n}\right)}^{p} e^{\mu t} d t \geq e^{\sum \mu_{i}(R-1)} \int_{R-1}^{R}\left\|\nabla_{e} f_{r}\right\|_{\mathbb{L}^{p}\left(\mathbb{T}^{n}\right)}^{p} d t .
$$

So we write

$$
e^{\sum \mu_{i}(R-1)} \int_{R-1}^{R}\left\|\nabla_{e} f_{r}\right\|_{\mathbb{L}^{p}\left(\mathbb{T}^{n}\right)}^{p} d t \leq e^{p \mu_{n} R} \int_{B(R) \backslash B(R-1)}|\nabla f|^{p} d V o l .
$$

Fixing $r \in[R-1, R]$, let us write Poincaré inequality on the torus for the function $f_{r}(\theta)$. There exists a number $c_{r}$ such that

$$
\int_{\mathbb{T}^{n}}\left|f_{r}(\theta)-c_{r}\right|^{p} d \theta \leq\left(C_{p}\left(\mathbb{T}^{n}\right)\right)^{p} \int_{\mathbb{T}^{n}}\left|\nabla_{e} f_{r}(\theta)\right|^{p} d \theta
$$

where $C_{p}\left(\mathbb{T}^{n}\right)$ is a Poincaré constant for $\mathbb{T}^{n}$. Next we consider the function $f_{r}(\theta)$ as a function on the ball $B(R)$ which does not depend on $t$. We integrate this inequality over $t$,

$$
\begin{aligned}
\int_{B(R)}\left|f_{r}(\theta)-c_{r}\right|^{p} d V o l & \leq\left(C_{p}\left(\mathbb{T}^{n}\right)\right)^{p} \int_{0}^{R} \int_{\mathbb{T}^{n}}\left|\nabla_{e} f_{r}(\theta)\right|^{p} d \theta e^{\sum \mu_{i} t} d t \\
& \leq \frac{e^{\sum \mu_{i} R}}{\sum \mu_{i}}\left(C_{p}\left(\mathbb{T}^{n}\right)\right)^{p} \int_{\mathbb{T}^{n}}\left|\nabla_{e} f_{r}(\theta)\right|^{p} d \theta .
\end{aligned}
$$

We integrate over $r$ from $R-1$ to $R$ and exploit inequality (4). It gives

$$
\int_{R-1}^{R}\left(\int_{B(R)}\left|f_{r}(\theta)-c_{r}\right|^{p} d V o l\right) d r \leq A(\mu)\left(C_{p}\left(\mathbb{T}^{n}\right)\right)^{p} e^{p \mu_{n} R} \int_{B(R) \backslash B(R-1)}|\nabla f|^{p} d V o l,
$$

where $A(\mu)$ is a constant which depends only on $\mu_{i}, i=1, \ldots, n$. Now we apply Hölder inequality again,

$$
\begin{aligned}
\int_{R-1}^{R}\left\|f_{r}-c_{r}\right\|_{\mathbb{L}^{p}(B(R))} d r & \leq\left(\int_{R-1}^{R} \int_{B(R)}\left|f_{r}-c_{r}\right|^{p} d V o l d r\right)^{1 / p} \\
& \leq\left(A(\mu)\left(C_{p}\left(\mathbb{T}^{n}\right)\right)^{p} e^{p \mu_{n} R} \int_{B(R) \backslash B(R-1)}|\nabla f|^{p} d V o l\right)^{1 / p} \\
& \leq(A(\mu))^{1 / p} C_{p}\left(\mathbb{T}^{n}\right) e^{\mu_{n} R}\|\nabla f\|_{\mathbb{L}^{p}(B(R))}
\end{aligned}
$$

Set $c=\int_{R-1}^{R} c_{r} d r$. In the following chain of inequalities we will first apply triangle inequality and then we will use the fact that the norm of the integral is less than or equal 
to the integral of the norm (briefly $\left\|\int f d r\right\|=\int\|f\| d r$ ).

$$
\begin{aligned}
\|f-c\|_{\mathbb{L}^{p}(B(R))} & =\left\|\int_{R-1}^{R}\left(f-c_{r}\right) d r\right\|_{\mathbb{L}^{p}(B(R))} \\
& \leq\left\|\int_{R-1}^{R}\left(f-f_{r}\right) d r\right\|_{\mathbb{L}^{p}(B(R))}+\left\|\int_{R-1}^{R}\left(f_{r}-c_{r}\right) d r\right\|_{\mathbb{L}^{p}(B(R))} \\
& \leq \int_{R-1}^{R}\left(\left\|f-f_{r}\right\|_{\mathbb{L}^{p}(B(R))}+\left\|f_{r}-c_{r}\right\|_{\mathbb{L}^{p}(B(R))}\right) d r \\
& \leq \frac{p}{\mu}\|\nabla f\|_{\mathbb{L}^{p}(B(R))}+(A(\mu))^{1 / p} C_{p}\left(\mathbb{T}^{n}\right) e^{\mu_{n} R}\|\nabla f\|_{\mathbb{L}^{p}(B(R))} .
\end{aligned}
$$

\section{LOWER BOUnd on Poincaré CONSTANT}

Let $Z_{\mu}$ denote $\mathbb{T}^{n} \times \mathbb{R}$ equipped with metrics $d t^{2}+\sum e^{2 \mu_{i} t} d x_{i}^{2}$, where we suppose $\mu_{1} \leq$ $\mu_{2} \leq \ldots \leq \mu_{n}$. Let $O, O^{\prime}=(0, \ldots, 0)$ be base points of $Z$ and $Z^{\prime}$ respectively. We notice that the "width" of $\mathbb{T}^{n} \times(-\infty, 0]$ is finite so it is at finite distance from a ray $(-\infty, 0]$, so from now on, we shall focus our attention on the part of $B_{Z}(O, R)$ where $t \geq 0$. Indeed, we want to consider quasi-isometric embeddings of balls $\mathbb{T}^{n} \times[-R, R]$. The volume of $T^{n} \times(-\infty, 0]$ is finite, whereas the volume of $\mathbb{T}^{n} \times[0, R]$ is exponential in $R$. Hence, only a negligible part of $\mathbb{T}^{n} \times[-R, R]$ can be sent to the negative part $\mathbb{T}^{n} \times(-\infty, 0]($ compare to subsection 3.1).

Consider a ball $B_{Z}(O, R)$ in $Z=Z_{\mu}$ and its quasi-isometric embedding in $Z^{\prime}=Z_{\mu^{\prime}}$. In this section we will give a lower bound for the sum of quasi-isometric constants $\lambda+c$ in function of $R$, using our results on transported Poincaré inequalities. We have to notice that our method does not apply to a general quasi-isometric embedding. We will consider only quasi-isometric embeddings which are homotopy equivalences.

Why do we want to consider these spaces $Z_{\mu}$ ? Following U.Hamenstädt [6] and $\mathrm{X}$. Xie [5], 4, there is a family of hyperbolic spaces whose quasi-isometric classification is known, that are spaces with transitive Lie groups of isometries. In this family (classified by E.Heintze [7]), the easiest spaces are $X_{\mu}$. We also know their $\mathbb{L}^{p}$ cohomologies (Pansu, [3]). Still they are rather difficult because their $\mathbb{L}^{p}$ cohomology vanishes for a delicate global reason, which is hard to make quantitative, on balls. Fortunately, their quotients $Z_{\mu}$ by $\mathbb{Z}^{n}$ are simpler. We can also say that the spaces $Z_{\mu}$ are hyperbolic spaces with ideal boundaries being products of circles supplied with power of the standard metric.

\subsection{Statement of theorems.}

Theorem 4. Let $Z, Z^{\prime}$ be two locally homogeneous hyperbolic metric spaces with metrics $d t^{2}+\sum e^{2 \mu_{i} t} d x_{i}^{2}$ and $d t^{2}+\sum e^{2 \mu_{i}^{\prime} t} d x_{i}^{2}$ respectively, $0<\mu_{1} \leq \mu_{2} \leq \ldots \leq \mu_{n}$ and $0<\mu_{1}^{\prime} \leq$ $\mu_{2}^{\prime} \leq \ldots \leq \mu_{n}^{\prime}$. Assume also that $\sum \mu_{i} / \mu_{n}>\sum \mu_{i}^{\prime} / \mu_{n}^{\prime}$. Suppose that there exist constants $a$ and $b$ such that for any $i, b \leq \mu_{i}, \mu_{i}^{\prime} \leq a$. Then there exists a constant $G_{0}(a, b)$ such that the following holds. Let $\Theta: B_{Z}(R) \rightarrow Z^{\prime}$ be a continuous $\left(\lambda_{1}, \lambda_{2}, c_{1}, c_{2}\right)$-quasi-isometric embedding, inducing an isomorphism on fundamental groups. Suppose that $\Theta$ sends base 
point to base point, $\Theta(O)=O^{\prime}$ and that $R \geq 8\left(\lambda_{1}+c_{1}\right)+\left(\lambda_{2}+c_{2}\right)+1$. If $p>\sum \mu_{i}^{\prime} / \mu_{n}^{\prime}$, up to replacing $Z$ with a connected 2 -sheeted covering, the Poincaré constant $C_{p}(\mu)$ for a ball of radius $R$ in the space $Z$ is bounded from below by

$$
C_{p}(\mu) \geq\left(G_{0}(a, b)\right)^{1 / p}\left(\lambda_{1}+c_{1}\right)^{-3 / p-2 / p^{2}} e^{-\left(9 / p+3 / p^{2}\right)\left(\lambda_{1}+c_{1}\right)} e^{\left(\sum \mu_{i} / p\right) R}\left(p-\sum \mu_{i}^{\prime} / \mu_{n}^{\prime}\right)^{1 / p} .
$$

This theorem is not symmetric, it can be applied only in one direction: it does not give any lower bound to the quasi-isometric embeddings of $Z_{\mu}$ to $Z_{\mu^{\prime}}$ and of $Z_{\mu^{\prime}}$ to $Z_{\mu}$ at the same time.

As we have already mentioned, we are able to treat the quantitative problem only for quasi-isometric embeddings which are homotopy equivalences. So we modify Definition 3 in the following way.

Definition 12. Let $X, Y$ be metric spaces, $x_{0}, y_{0}$ their base points respectively. The homotopy quasi-isometric distortion growth is the function

$$
\begin{array}{r}
D_{h G}\left(X, x_{0}, Y, y_{0}\right)(R)=\inf \left\{d \mid \exists f: B_{X}\left(x_{0}, R\right) \rightarrow Y \text { a }\left(\lambda_{f}, c_{f}\right)\right. \text {-quasi-isometric embedding } \\
\text { such that } \left.f\left(x_{0}\right)=y_{0} \text { and } f \text { is a homotopy equivalence, } d=\lambda_{f}+c_{f}\right\} .
\end{array}
$$

Theorem 5. Let $Z, Z^{\prime}$ be two locally homogeneous hyperbolic metric spaces with metrics $d t^{2}+\sum e^{2 \mu_{i} t} d x_{i}^{2}$ and $d t^{2}+\sum e^{2 \mu_{i}^{\prime} t} d x_{i}^{2}$ respectively, $0<\mu_{1} \leq \mu_{2} \leq \ldots \leq \mu_{n}$ and $0<$ $\mu_{1}^{\prime} \leq \mu_{2}^{\prime} \leq \ldots \leq \mu_{n}^{\prime}$. Assume also that $\sum \mu_{i} / \mu_{n}>\sum \mu_{i}^{\prime} / \mu_{n}^{\prime}$. Suppose that there exist constants $a$ and $b$ such that for any $i b \leq \mu_{i}, \mu_{i}^{\prime} \leq a$. Then there exist constants $G_{1}(a, b)$ and $G_{2}(a, b)$ such that the following holds. The homotopy distortion growth (see Definition 12) for quasi-isometrical embedding of $B_{Z}(R)$ into $Z^{\prime}$ is bounded from below by

$$
D_{h G}(R) \geq \min \left\{G_{1}\left(\frac{\sum \mu_{i}}{\mu_{n}}-\frac{\sum \mu_{i}^{\prime}}{\mu_{n}^{\prime}}\right) R-G_{2}, \frac{1}{8} R\right\} .
$$

Theorem 4 plays an important role in the proof of Theorem 5. Before proving these two theorems, we will discuss the double cover of the family of spaces under consideration and we will give some preliminary lemmas.

7.2. Lifting to a double covering space. Let us introduce a double covering of $Z^{\prime}$. Let $\tilde{Z}^{\prime}=\mathbb{R}^{n-1} / \mathbb{Z}^{n-1} \times \mathbb{R} / 2 \mathbb{Z} \times[0,+\infty)$ with the metric defined by the same formula as for $Z^{\prime}$ : $d t^{2}+\sum e^{2 \mu_{i} t} d x_{i}^{2}$. Consider the map $\tilde{Z}^{\prime} \rightarrow Z^{\prime}$ defined by

$$
\left(x_{1}, x_{2}, \ldots, x_{n}, t\right) \mapsto\left(x_{1}, x_{2}, \ldots, x_{n} \bmod 1, t\right) .
$$

So we identify $\left(x_{1}, x_{2}, \ldots, x_{n}, t\right)$ and $\left(x_{1}, x_{2}, \ldots, x_{n}+1, t\right)$ in $\tilde{Z}^{\prime}$. Consider a complex function $u\left(x_{1}, x_{2}, \ldots, x_{n}, t\right)=e^{\pi i x_{n}}$ on $\tilde{Z}^{\prime}$.

Composition of $u$ with the deck transformation $\iota^{\prime}: \tilde{Z}^{\prime} \rightarrow \tilde{Z}^{\prime}$

$$
\iota^{\prime}:\left(x_{1}, x_{2}, \ldots, x_{n}, t\right) \mapsto\left(x_{1}, x_{2}, \ldots, x_{n}+1, t\right)
$$

gives $u \circ \iota^{\prime}=-u$. 
By assumption, $\Theta: Z \rightarrow Z^{\prime}$ is a continuous map inducing an isomorphism on fundamental groups, and we have $\tilde{Z}^{\prime}$ which is a covering space of $Z^{\prime}$. We need to show that there exists a non-trivial covering space $\tilde{Z} \rightarrow Z$ such that the following diagram commutes.

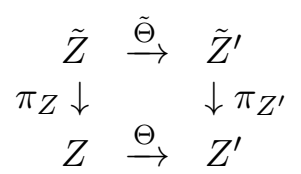

Define

$$
\tilde{Z}=\left\{\left(z, \tilde{z}^{\prime}\right) \mid z \in Z, \tilde{z}^{\prime} \in \pi_{Z^{\prime}}^{-1}(\Theta(z))\right\},
$$

that is $\tilde{Z} \subset Z \times \tilde{Z}^{\prime}$. Let $\left[\gamma^{\prime}\right]$ be a loop in $Z^{\prime}$ which does not lift to a loop in $\tilde{Z}^{\prime}$. By hypothesis, there exists a loop $\gamma$ in $Z$ such that $\Theta(\gamma)$ is homotopic to $\gamma^{\prime}$. Then $\gamma$ does not lift to a loop in $\tilde{Z}$. There exists an isometry $\iota$ of order 2 on $\tilde{Z}$ such that $\tilde{\Theta} \circ \iota=\iota^{\prime} \circ \tilde{\Theta}$.

7.3. Lifting of $\Theta$. Here we will prove that in the constructed double coverings $\Theta$ lifts to a map satisfying the right-hand inequality in the definition of quasi-isometry with constants $\lambda_{1}$ and $2 c_{1}$. We need two preliminary lemmas concerning distances in two-fold coverings.

Lemma 6. Let $Z=Z_{\mu}$ be a locally homogeneous space. There is an effective constant $c_{0}(\mu)$ with the following effect. Let $z$ be a point in $Z$ in the region where $t \geq c_{0}$. Let $c=t(z)$. Every loop of length less than $c$ based at $z$ is null-homotopic.

Proof. Let $\pi_{s}: Z \rightarrow T^{n} \times\{s\} \subset Z$ denotes projection onto the first factor. This is a homotopy equivalence. Note that $\pi_{s}$ is length decreasing on $\{(t, x) \in Z ; t \geq s\}$. Moreover, on $T^{n} \times\{t\}, \pi_{s}$ decreases length by $e^{\mu_{1}(s-t)}$ at least. Let $\gamma$ be a non null-homotopic geodesic loop at $z$. Assume that its length is $\leq 2 c$. Then $\gamma \subset\left\{(t, x) \in Z ; t \geq \frac{c}{2}\right\}$, therefore

$$
\operatorname{length}\left(\pi_{\frac{c}{2}}(\gamma)\right) \leq c
$$

thus

$$
\text { length }\left(\pi_{0}(\gamma)\right) \leq c e^{-\mu_{1} \frac{c}{2}}
$$

Since $\pi_{0}(\gamma)$ is not null-homotopic, its length is at least 1 , and this shows that

$$
c \geq e^{\mu_{1} \frac{c}{2}} .
$$

This can happen only for $c \leq c_{0}\left(\mu_{1}\right)$.

Lemma 7. Let $z_{1}, z_{2}$ be two points in $Z$ such that $d\left(O^{\prime}, \Theta\left(z_{1}\right)\right)>c_{1}$ or $d\left(O^{\prime}, \Theta\left(z_{2}\right)\right)>c_{1}$ and $d\left(z_{1}, z_{2}\right) \leq c_{1} / \lambda_{1}$. Then $d\left(\tilde{\Theta}\left(\tilde{z}_{1}\right), \tilde{\Theta}\left(\tilde{z}_{2}\right)\right)=d\left(\Theta\left(z_{1}\right), \Theta\left(z_{2}\right)\right)$.

Proof. Let $\tilde{z}_{1} \in \tilde{Z}$ be such that $d\left(\tilde{O}, \tilde{z}_{1}\right)>c_{1}$. Set

$$
\begin{aligned}
W & =\left\{\tilde{z}_{2} \in \tilde{Z} \mid, d\left(\tilde{z}_{1}, \tilde{z}_{2}\right) \leq c_{1}\right\}, \\
U & =\left\{\tilde{z}_{2} \in W \mid d\left(\tilde{\Theta}\left(\tilde{z}_{1}\right), \tilde{\Theta}\left(\tilde{z}_{2}\right)\right)=d\left(\Theta\left(z_{1}\right), \Theta\left(z_{2}\right)\right)\right\} \subset W, \\
V & =\left\{\tilde{z}_{2} \in W \mid d\left(\tilde{\Theta}\left(\tilde{z}_{1}\right), \iota^{\prime} \circ \tilde{\Theta}\left(\tilde{z}_{2}\right)\right)=d\left(\Theta\left(z_{1}\right), \Theta\left(z_{2}\right)\right)\right\} \subset W .
\end{aligned}
$$


By construction, $W=U \cup V$. Let us show that the intersection of $U$ and $V$ is empty

$$
U \cap V=\left\{\tilde{z}_{2} \in W \mid d\left(\tilde{\Theta}\left(\tilde{z}_{1}\right), \iota^{\prime} \circ \tilde{\Theta}\left(\tilde{z}_{2}\right)\right)=d\left(\tilde{\Theta}\left(\tilde{z}_{1}\right), \tilde{\Theta}\left(\tilde{z}_{2}\right)\right)\right\} .
$$

If $\tilde{z}_{2} \in U \cap V$, then the geodesic segments connecting $\tilde{\Theta}\left(\tilde{z}_{1}\right)$ with $\tilde{\Theta}\left(\tilde{z}_{2}\right)$ and $\tilde{\Theta}\left(\tilde{z}_{1}\right)$ with $\iota^{\prime} \circ \tilde{\Theta}\left(\tilde{z}_{2}\right)$ induce a loop $\gamma$ in $Z^{\prime}$ of length $2 d\left(\Theta\left(z_{1}\right), \Theta\left(z_{2}\right)\right) \leq 2\left(\lambda_{1}\left(c_{1} / \lambda_{1}\right)+c_{1}\right)=4 c_{1}$ which is not homotopic to 0 . According to Lemma 6, this is incompatible with the assumption that $d\left(O^{\prime}, \Theta\left(z_{1}\right)\right)>c_{1}$. Hence, $U \cap V$ is empty. Since $U$ is non-empty (it contains at least $\tilde{z}_{1}$ ) and closed in $W, V$ is closed in $W$ and $W$ is connected, we conclude that $U=W$, which finishes the proof.

Lemma 8. $A\left(\lambda_{1}, \lambda_{2}, c_{1}, c_{2}\right)$-quasi-isometric embedding $\Theta: Z \rightarrow Z^{\prime}$ lifts to a "quasiLipschitz" map $\tilde{\Theta}: \tilde{Z} \rightarrow \tilde{Z}^{\prime}$, that is, for any two points $\tilde{z}_{1}, \tilde{z}_{2} \in \tilde{Z}$,

$$
d\left(\tilde{\Theta}\left(\tilde{z}_{1}\right), \tilde{\Theta}\left(\tilde{z}_{2}\right)\right) \leq \lambda_{1} d\left(\tilde{z}_{1}, \tilde{z}_{2}\right)+2 c_{1}
$$

Proof. Let $\tilde{\gamma} \subset \tilde{Z}$ be a geodesic between $\tilde{z}_{1}$ and $\tilde{z}_{2}$. Let $t_{1}$ be the first point such that $d\left(\tilde{\Theta} \gamma(t), \tilde{O}^{\prime}\right) \leq c_{1}$ and $t_{2}$ be the last point with such a property (if such points $t_{1}, t_{2}$ do not exist, then we can apply the following arguments directly to $d\left(\tilde{\Theta}\left(\tilde{z}_{1}\right), \tilde{\Theta}\left(\tilde{z}_{2}\right)\right)$ instead of cutting the curve in three parts and considering $\left.d\left(\tilde{\Theta}\left(\tilde{z}_{1}\right), \tilde{\Theta} \tilde{\gamma}\left(t_{1}\right)\right)+d\left(\tilde{\Theta}\left(\tilde{z}_{1}\right), \tilde{\Theta} \tilde{\gamma}\left(t_{2}\right)\right)\right)$. Then

$$
d\left(\tilde{\Theta}\left(\tilde{z}_{1}\right), \tilde{\Theta}\left(\tilde{z}_{2}\right)\right) \leq d\left(\tilde{\Theta} \tilde{\gamma}\left(t_{1}\right), \tilde{\Theta} \tilde{\gamma}\left(t_{2}\right)\right)+d\left(\tilde{\Theta}\left(\tilde{z}_{1}\right), \tilde{\Theta} \tilde{\gamma}\left(t_{1}\right)\right)+d\left(\tilde{\Theta}\left(\tilde{z}_{1}\right), \tilde{\Theta} \tilde{\gamma}\left(t_{2}\right)\right)
$$

By definition of $t_{1}$ and $t_{2}, d\left(\tilde{\Theta} \tilde{\gamma}\left(t_{1}\right), \tilde{\Theta} \tilde{\gamma}\left(t_{2}\right)\right) \leq 2 c_{1}$. Now divide parts of $\gamma$ between $\tilde{\Theta}\left(\tilde{z}_{1}\right)$ and $\tilde{\Theta} \tilde{\gamma}\left(t_{1}\right)$ and between $\tilde{\Theta}\left(\tilde{z}_{1}\right)$ and $\tilde{\Theta} \tilde{\gamma}\left(t_{2}\right)$ by segments of length $c_{1} / \lambda_{1}$. We apply the previous lemma to them, so

$$
d\left(\tilde{\Theta}\left(\tilde{z}_{1}\right), \tilde{\Theta} \tilde{\gamma}\left(t_{1}\right)\right)+d\left(\tilde{\Theta}\left(\tilde{z}_{1}\right), \tilde{\Theta} \tilde{\gamma}\left(t_{2}\right)\right) \leq N\left(\lambda_{1} \frac{c_{1}}{\lambda_{1}}+c_{1}\right),
$$

where $N \leq d\left(\tilde{z}_{1}, \tilde{z}_{2}\right) /\left(c_{1} / \lambda_{1}\right)$ is the number of segments in the subdivision. So,

$$
d\left(\tilde{\Theta}\left(\tilde{z}_{1}\right), \tilde{\Theta}\left(\tilde{z}_{2}\right)\right) \leq 2 c_{1}+2 \lambda_{1} d\left(\tilde{z}_{1}, \tilde{z}_{2}\right) .
$$

7.4. Proof of Theorem 4 - Part 1. Let $\psi^{\prime}$ be a kernel on $\tilde{Z}$ which is invariant by isometry, that is, for any isometry $\iota$,

$$
\psi^{\prime}\left(\iota\left(\tilde{z}_{1}\right), \iota\left(\tilde{z}_{2}\right)\right)=\psi^{\prime}\left(\tilde{z}_{1}, \tilde{z}_{2}\right) .
$$

As an example of such a kernel we can consider a kernel depending only on the distance between points. Let $\zeta$ be a kernel on $\tilde{Z}^{\prime}$ which is also invariant by isometries. Define a complex function $v$ on $\tilde{Z}$ as follows

$$
v(\cdot)=\left(\int_{Y} u\left(\tilde{z}^{\prime}\right) \zeta\left(\tilde{\Theta}(\tilde{z}), \tilde{z}^{\prime}\right) d \tilde{z}^{\prime}\right) * \psi^{\prime}(\cdot, \tilde{z}) .
$$

We will write shortly for the integral

$$
u *_{t} \zeta(\tilde{\Theta})(\tilde{z})=\int_{Y} u\left(\tilde{z}^{\prime}\right) \zeta\left(\tilde{\Theta}(\tilde{z}), \tilde{z}^{\prime}\right) d \tilde{z}^{\prime}
$$


Then $v \circ \iota=-v$. Indeed,

$$
v \circ \iota=\left(u *_{t} \zeta(\tilde{\Theta})\right) * \psi^{\prime} \circ \iota=\left(u *_{t} \zeta(\tilde{\Theta}) \circ \iota\right) * \psi^{\prime} .
$$

On the other hand, using both relations $\tilde{\Theta} \circ \iota=\iota^{\prime} \circ \tilde{\Theta}$ and $\left(\iota^{\prime}\right)^{2}=i d$, we have

$$
\begin{array}{r}
u *_{t} \zeta(\tilde{\Theta}) \circ \iota(\tilde{z})=\int u\left(\tilde{z}^{\prime}\right) \zeta\left(\tilde{\Theta}(\iota \tilde{z}), \tilde{z}^{\prime}\right) d \tilde{z}^{\prime}=\int u\left(\tilde{z}^{\prime}\right) \zeta\left(\iota^{\prime} \tilde{\Theta}(\tilde{z}),\left(\iota^{\prime}\right)^{2} \tilde{z}^{\prime}\right) d \tilde{z}^{\prime}= \\
=\int u\left(\tilde{z}^{\prime}\right) \zeta\left(\tilde{\Theta}(\tilde{z}), \iota^{\prime} \tilde{z}^{\prime}\right) d \tilde{z}^{\prime}=\int u\left(\iota^{\prime} \tilde{z}^{\prime}\right) \zeta\left(\tilde{\Theta}(\tilde{z}), \tilde{z}^{\prime}\right) d \tilde{z}^{\prime}=-u *_{t} \zeta(\tilde{\Theta}),
\end{array}
$$

hence $v$ is skewsymmetric with respect to $\iota$. We get immediately that $\int v=0$. Now we apply successively Lemma 3 and Lemma 4 .

Step 1. By Lemma 3 there exists a kernel $\psi_{1}$ on $\tilde{Z}$ which is controlled by $a$ and $b$ and such that

$$
\left(\int\left|\nabla\left(u *_{t} \zeta(\tilde{\Theta}) * \psi^{\prime}\right)\right|^{p}\right)^{1 / p} \leq N_{\psi_{1}}\left(u *_{t} \zeta(\tilde{\Theta})\right),
$$

where for $\psi_{1}$ we have the width of support is $R^{\psi_{1}}=R^{\psi^{\prime}}$ and

$$
\sup \psi_{1} \leq \frac{\sup \nabla \psi^{\prime} \sup \psi^{\prime}}{\inf _{z} \operatorname{Vol} B\left(\tilde{z}, R^{\psi}\right)}
$$

Step 2. By Lemma 4 there exists a kernel $\zeta_{1}$ on $\tilde{Z}^{\prime}$ such that

$$
N_{\psi_{1}}\left(u *_{t} \zeta(\tilde{\Theta})\right) \leq \tilde{C} N_{\zeta_{1}}(u)
$$

where the width of support of $\zeta_{1}$ is $2 R^{\zeta}+\lambda_{1} R^{\psi^{\prime}}+c_{1}$, the supremum of $\zeta_{1}$ is

$$
\sup \zeta_{1}=\frac{\sup \psi_{1}}{c_{\tau}^{Y}} e^{2 R^{\zeta}+\lambda_{1} R^{\psi^{\prime}}+c_{1}}\left(2 \lambda_{1} R^{\zeta}+c_{1}\right)^{2}
$$

and

$$
\tilde{C}=\frac{1}{c_{\tau}^{Y}}\left(\sup \psi_{1}\right)^{3 / p} e^{\left(\left(2+\lambda_{1}\right) R^{\psi^{\prime}}+c_{1}\right) / p}\left(\left(2+\lambda_{1}\right) R^{\psi^{\prime}}+c_{1}\right)^{2 / p} .
$$

Step 3. Applying Lemma 3 we get that there exists a kernel $\zeta_{2}$ on $\tilde{Z}^{\prime}$ such that

$$
N_{\zeta_{2}}(u) \leq C(n)\|\nabla u\|_{p}
$$

we remind that the constant $C(n)$ depends only on the dimension of $\tilde{Z}^{\prime}$ if the Ricci curvature is bounded from below, that is $\sup \mu_{i}$ is bounded.

Step 4 . Here we merely need to pass from $N_{\zeta_{1}}$ to $N_{\zeta_{2}}$. We apply Lemma 3 once more

$$
N_{\zeta_{1}} \leq \hat{C} N_{\zeta_{2}}
$$

where

$$
\hat{C}=\frac{\sup \zeta_{1} \sup \zeta_{2}}{c_{\tau}^{Y}} \frac{R^{\zeta_{2}}}{\varepsilon^{\zeta_{2}}}(2 e)^{\left(2 R^{\zeta}+\lambda_{1} R^{\psi^{\prime}}+c_{1}\right) / \varepsilon^{\zeta_{2}}}
$$


Choose $\psi^{\prime}$ and $\zeta$ such that $R^{\psi^{\prime}}=1$ and $R^{\zeta}=1$. Then $\sup \psi^{\prime}$ and $\sup \zeta$ are controlled by $a$ and $b$. We note also that $\varepsilon^{\zeta_{2}}=1$. So combining all inequalities we get

$$
\int_{B(R)}|\nabla v|^{p} \leq C_{1}(a, b)\left(\lambda_{1}+c_{1}\right)^{3+2 / p} e^{(9+3 / p)\left(\lambda_{1}+c_{1}\right)} \int_{\mathbb{T}^{n} \times[0,+\infty]}|\nabla u|^{p},
$$

where $C_{1}(a, b)$ is a constant depending only on $a, b$ and dimension $n$. Let $Q=\lambda_{1}+c_{1}$ and

$$
C(Q)=\left(\lambda_{1}+c_{1}\right)^{3+2 / p} e^{(9+3 / p)\left(\lambda_{1}+c_{1}\right)} .
$$

7.5. Proof of Theorem 4. We will give a lower bound for the $\mathbb{L}^{p}$-norm of the function $v=(u * \phi) * \psi^{\prime}$. Our aim is to prove that the absolute value of $v$ is nearly constant. For simplicity of notations we suppose first that the volume growth of $Z_{\mu}$ and $Z_{\mu^{\prime}}$ is the same, that is $\sum \mu_{i}=\sum \mu_{i}^{\prime}$. We will write $|\mu|$ and $\left|\mu^{\prime}\right|$ for these sums respectively. We are going to show that there exists a subset $A$ of the ball $B\left(z_{0}, R\right)$ such that on the one hand the volume of $A$ is rather big, that is $\operatorname{Vol}(A) \geq \operatorname{Vol}\left(B\left(z_{0}, R\right)\right) / 2$ and on the other hand its image lies rather far from the base point $\Theta(A) \cap B\left(z_{0}^{\prime}, R-\left(\lambda_{1}+c_{1}+\lambda_{2}+c_{2}\right)\right)=\emptyset$.

Denote by $r=\lambda_{2}+c_{2}$. We will construct a finite subset $J$ in $B\left(z_{0}, R\right) \subset Z_{\mu}$ and a partition of $J$ into $e^{|\mu| r}$ subsets $\left\{J_{k}\right\}_{k=1, \ldots, n}$, each of cardinality $\left|J_{k}\right|=e^{|\mu|(R-r)}$ with the following property

- (P) For any $k \in\{1, \ldots, n\}$ if $z_{1}$ and $z_{2}$ are points of $J_{k}$ then the open balls of radius $r$ centered at these points are disjoint.

So, let $z_{1}, z_{2} \in J_{k}$ be two different points. It follows from (P) that

$$
2 r \leq d\left(z_{1}, z_{2}\right) \leq \lambda_{2} d\left(\Theta\left(z_{1}\right), \Theta\left(z_{2}\right)\right)+c_{2},
$$

hence $d\left(\Theta\left(z_{1}\right), \Theta\left(z_{2}\right)\right) \geq 2$, so the balls $B\left(\Theta\left(z_{1}\right), 1\right)$ and $B\left(\Theta\left(z_{2}\right), 1\right)$ are disjoint. Fix some $d>0$ and denote by $J_{k}^{\prime} \subset J_{k}$ the set of points whoes images are not farther than $R-d$ from $z_{0}^{\prime}$ that is if $z \in J_{k}^{\prime}$ then $d\left(z_{0}^{\prime}, \Theta(z)\right) \leq R-d$. We obtain

$$
\left|J_{k}^{\prime}\right| \operatorname{Vol}(B(\Theta(z), 1)) \leq \operatorname{Vol}\left(B\left(z_{0}^{\prime}, R-d+1\right)\right),
$$

and we conclude that $\left|J_{k}^{\prime}\right| \leq e^{|\mu|(R-d)}$. Denote the union of $J_{k}^{\prime}$ by $J^{\prime}$ then $\left|J^{\prime}\right| \leq e^{|\mu|(R-d+r)}$. Hence, whenever $d \geq r+1$,

$$
\frac{\left|J^{\prime}\right|}{|J|} \leq e^{|\mu|(r-d)} \leq \frac{1}{2}
$$

So, we choose $d=r+1$. Now let $A$ be the union of all 1-balls centered at points of $J \backslash J^{\prime}$, $A=\cup_{z \in J \backslash J^{\prime}} B(z, 1)$. The volume $\operatorname{Vol} A \geq 1 / 2 \operatorname{Vol}\left(B\left(z_{0}, R\right)\right)$. By definition of $A$, for any point $z \in A$ there exists a point $z^{\prime} \in J \backslash J^{\prime}$ at most 1 -far away from $z, d\left(z, z^{\prime}\right) \leq 1$. Applying triangle inequality we get $d\left(z_{0}^{\prime}, \Theta(z)\right) \geq d\left(z_{0}^{\prime}, \Theta\left(z^{\prime}\right)\right)-\left(\lambda_{1}+c_{1}\right) \geq R-\left(\lambda_{1}+c_{1}+\lambda_{2}+c_{2}\right)$.

Here we describe the set $J \subset\{R\} \times \mathbb{R}^{n} / \mathbb{Z}^{n}$ (we fix the first coordinate $t=R$ ). This is the set of points $z=\left(R, x_{1}, \ldots, x_{n}\right)$ such that for any $i=1, \ldots, n, x_{i}$ is an integer multiple of $e^{-\mu_{i} R}$ modulo 1 . $J_{0}$ is the subset of points such that for any $i, x_{i}$ is a whole multiple of $e^{\mu_{i}(r-R)}$. Let $K$ be the set of vectors $k=\left(0, k_{1}, \ldots, k_{n}\right)$ such that for any $i$ the number 
$e^{\mu_{i} R} k_{i}$ is an integer between 0 and $e^{\mu_{i}(r-R)}-1$. For $k \in K$, we define $J_{k}=J_{0}+k$. Then for any two different points $z_{1}, z_{2}$ of $J_{k}$,

$$
d\left(z_{1}, z_{2}\right)=\max \log \left(\left|x_{i}^{1}-x_{i}^{2}\right|^{1 / \mu_{i}}\right) \geq r .
$$

We constructed the needed set. Now we notice that the lifting $\tilde{A} \subset \tilde{Z}$ of $A$ has the same properties relatively to $\tilde{\Theta}$ : the image $\tilde{\Theta}(\tilde{A})$ lies at distance at least $R-\left(\lambda_{1}+c_{1}+\lambda_{2}+c_{2}\right)$ from the base point and the volume of $\tilde{A}$ is at least a half of the volume of the ball $B\left(\tilde{z}_{0}, R\right)$.

Now let us compute $|v(\tilde{z})|$ for $\tilde{z} \in \tilde{A}$ (in fact here we will give an upper bound for $|v|$ which is true for all $\tilde{z} \in B\left(z_{0}, R\right)$ and a lower bound for $\left.\tilde{z} \in \tilde{A}\right)$. We remind that by construction, $\tilde{z}$ is sent far from the base point, $d\left(\tilde{z}_{0}^{\prime}, \tilde{\Theta}(\tilde{z})\right) \geq R-\left(\lambda_{1}+c_{1}+\lambda_{2}+c_{2}\right)$.

$$
\begin{aligned}
\left|(u * \phi) * \psi^{\prime}(\tilde{z})\right|= & \left|\int_{X} \int_{Y} u\left(\tilde{z}^{\prime}\right) \zeta\left(\tilde{\Theta}\left(\tilde{z}_{1}\right), \tilde{z}^{\prime}\right) \psi^{\prime}\left(\tilde{z}, \tilde{z}_{1}\right) d \tilde{z}^{\prime} d \tilde{z}_{1}\right| \\
\geq & \left|\int_{X} \int_{Y}\left(u\left(\tilde{z}^{\prime}\right)-u(\tilde{\Theta}(\tilde{z}))+u(\tilde{\Theta}(\tilde{z}))\right) \zeta\left(\tilde{\Theta}\left(\tilde{z}_{1}\right), \tilde{z}^{\prime}\right) \psi^{\prime}\left(\tilde{z}, \tilde{z}_{1}\right) d \tilde{z}^{\prime} d \tilde{z}_{1}\right| \\
\geq & \left|\int_{X} \int_{Y}(u(\tilde{\Theta}(\tilde{z}))) \zeta\left(\tilde{\Theta}\left(\tilde{z}_{1}\right), \tilde{z}^{\prime}\right) \psi^{\prime}\left(\tilde{z}, \tilde{z}_{1}\right) d \tilde{z}^{\prime} d \tilde{z}_{1}\right| \\
& -\left|\int_{X} \int_{Y}\left(u\left(\tilde{z}^{\prime}\right)-u(\tilde{\Theta}(\tilde{z}))\right) \zeta\left(\tilde{\Theta}\left(\tilde{z}_{1}\right), \tilde{z}^{\prime}\right) \psi^{\prime}\left(\tilde{z}, \tilde{z}_{1}\right) d \tilde{z}^{\prime} d \tilde{z}_{1}\right| \\
\geq & 1-\int_{X} \int_{Y}\left|u\left(\tilde{z}^{\prime}\right)-u(\tilde{\Theta}(\tilde{z}))\right| \zeta\left(\tilde{\Theta}\left(\tilde{z}_{1}\right), \tilde{z}^{\prime}\right) \psi^{\prime}\left(\tilde{z}, \tilde{z}_{1}\right) d \tilde{z}^{\prime} d \tilde{z}_{1} .
\end{aligned}
$$

For the last inequality we shall use the following facts: $|u|=1$ and the integral of a kernel over the second argument is equal to 1 .

$$
\begin{aligned}
& \left|\int_{X} \int_{Y} u(\tilde{\Theta}(\tilde{z})) \zeta\left(\tilde{\Theta}\left(\tilde{z}_{1}\right), \tilde{z}^{\prime}\right) \psi^{\prime}\left(\tilde{z}, \tilde{z}_{1}\right) d \tilde{z}^{\prime} d \tilde{z}_{1}\right| \\
= & \left|\int_{X} u(\tilde{\Theta}(\tilde{z})) \psi^{\prime}\left(\tilde{z}, \tilde{z}_{1}\right)\left(\int_{Y} \zeta\left(\tilde{\Theta}\left(\tilde{z}_{1}\right), \tilde{z}^{\prime}\right) d \tilde{z}^{\prime}\right) d \tilde{z}_{1}\right| \\
= & \left|\int_{X} u(\tilde{\Theta}(\tilde{z})) \psi^{\prime}\left(\tilde{z}, \tilde{z}_{1}\right) d \tilde{z}_{1}\right|=|u(\tilde{\Theta}(\tilde{z}))|=1 .
\end{aligned}
$$

We need to estimate the double integral in Eq. (5) . $\psi^{\prime}\left(\tilde{z}, \tilde{z}_{1}\right)$ is non-zero if $d\left(\tilde{z}, \tilde{z}_{1}\right) \leq$ $R^{\psi^{\prime}}=1$ and $\zeta\left(\tilde{\Theta}\left(\tilde{z}_{1}\right), \tilde{z}^{\prime}\right)$ is non-zero if $d\left(\tilde{z}^{\prime}, \tilde{\Theta}\left(z_{1}\right)\right) \leq R^{\zeta}=1$. So the diameter of the set $\hat{S}$ of points $\tilde{z}^{\prime}$ such that the integrand is non-zero, is at most $2 \lambda_{1}+c_{1}+2 \leq 4\left(\lambda_{1}+c_{1}\right)$ because $\lambda_{1} \geq 1$. Hence $\hat{S}$ is contained in a ball $B_{\hat{S}}$ of radius $4\left(\lambda_{1}+c_{1}\right)$. Assume $\hat{z}^{\prime}=\tilde{\Theta}(\tilde{z}) \in \hat{S}$. Then by the mean value theorem, for any point $\tilde{z}^{\prime} \in \hat{S}$,

$$
\begin{aligned}
& \mid u\left(\tilde{z}^{\prime}\right)- u\left(\hat{z}^{\prime}\right)|\leq| \tilde{z}^{\prime}-\hat{z}^{\prime}\left|\sup _{\tilde{z}^{\prime} \in B_{\hat{S}}}\right| \nabla u\left(\tilde{z}^{\prime}\right)\left|\leq 8\left(\lambda_{1}+c_{1}\right) \sup _{\tilde{z}^{\prime} \in B_{\hat{S}}}\right| \frac{\partial u}{\partial \tilde{x}_{n}} \mid e^{-\mu_{n}^{\prime} t} \leq 8 \pi\left(\lambda_{1}+c_{1}\right) e^{-\mu_{n}^{\prime} t} \\
& \leq 8 \pi\left(\lambda_{1}+c_{1}\right) \sup _{\hat{z}^{\prime} \in B_{\hat{S}}} e^{-\mu_{n}^{\prime} d\left(O^{\prime}, \hat{z}^{\prime}\right)} \leq 8 \pi\left(\lambda_{1}+c_{1}\right) e^{-\mu_{n}^{\prime}\left(R-\left(\lambda_{1}+c_{1}+\lambda_{2}+c_{2}\right)-2\left(\lambda_{1}+c_{1}\right)\right)} \leq \frac{1}{2}
\end{aligned}
$$


for $R \geq 8\left(\lambda_{1}+c_{1}\right)+\left(\lambda_{2}+c_{2}\right)=R_{0}$. Hence we have proved that

$$
\begin{aligned}
& \frac{1}{2} \leq\left|(u * \phi) * \psi^{\prime}(\tilde{z})\right| \quad \text { if } \quad \tilde{z} \in \tilde{A} \\
& \left|(u * \phi) * \psi^{\prime}(\tilde{z})\right| \leq 1 \quad \text { if } \quad \tilde{z} \in B\left(\tilde{z}_{0}, R\right) .
\end{aligned}
$$

And we conclude from this relation that for $R \geq R_{0}+1$,

$$
\int_{B(R)}|v|^{p} \geq \frac{1}{2^{p}} \operatorname{Vol}(B(R))-\operatorname{Vol}\left(B\left(R_{0}\right)\right) \geq e^{\left(\sum \mu_{i}\right) R} / 2^{p+1} .
$$

Let us compute the integral $\int|\nabla u|^{p}$.

$$
\int|\nabla u|^{p}=\int\left|\frac{\partial u}{\partial x_{n}}\right|^{p} e^{-\mu_{n}^{\prime} p t} e^{\left(\sum \mu_{i}^{\prime}\right) t} d t d x_{n}=\pi \int_{0}^{+\infty} e^{\left(\sum \mu_{i}^{\prime}-p \mu_{n}^{\prime}\right) t} d t=\frac{\mu_{n}^{\prime} \pi}{-\sum \mu_{i}^{\prime} / \mu_{n}^{\prime}+p} .
$$

Hence the Poincaré constant $C_{p}(\mu)$ for $Z$ satisfies

$$
\begin{aligned}
\left(C_{p}(\mu)\right)^{p} & \geq \frac{\|v\|^{p}}{\|\nabla v\|^{p}} \geq \frac{\|v\|^{p}}{C_{1}(a, b) C(Q)\|\nabla u\|^{p}} \\
& \geq\left(\mu_{n}^{\prime} \pi 2^{p+1} C_{1}(a, b) C(Q)\right)^{-1} e^{\left(\sum \mu_{i}\right) R}\left(p-\sum \mu_{i}^{\prime} / \mu_{n}^{\prime}\right) .
\end{aligned}
$$

This proves the claim in Theorem 4 .

7.6. Proof of Theorem 5. Let $\Theta: B_{Z}(R) \rightarrow Z^{\prime}$ be a $\left(\lambda_{1}, \lambda_{2}, c_{1}, c_{2}\right)$-quasi-isometric embedding. By hypothesis, $\Theta$ is isomorphic on fundamental groups. Lemma 6 implies that $\Theta$ moves the origin a bounded distance away. Indeed, a non null-homotopic loop of length 1 based at $O$ is mapped to a non null-homotopic loop of length $\leq Q=\lambda_{1}+c_{1}$ based at $\Theta(O)$. This implies that $t(\Theta(O)) \leq 4 Q$ and $d\left(O^{\prime}, \Theta(O)\right) \leq 4 Q+1$.

The space $\tilde{Z}$ is of the form $\tilde{T} \times \mathbb{R}$ where $\tilde{T} \rightarrow T$ is a connected 2-sheeted covering space of torus, that is $\tilde{T}$ is also a torus. Hence we can apply Theorem 3 , We have $C_{p}(\mu) \leq C_{2}(a, b) e^{\mu_{n} R}$. If $R \leq 8\left(\lambda_{1}+c_{1}\right)+\left(\lambda_{2}+c_{2}\right)$ there is nothing to prove. Otherwise we arrive at

$$
\left(\mu_{n}^{\prime} \pi 2^{p+1} C_{1}(a, b) C(Q)\right)^{-1 / p} e^{\left(\sum \mu_{i} / p\right) R}\left(p-\sum \mu_{i}^{\prime} / \mu_{n}^{\prime}\right)^{1 / p} \leq C_{2}(a, b) e^{\mu_{n} R} .
$$

Hence with $C_{3}(a, b)=\left(\mu_{n}^{\prime} \pi 2^{p+1} C_{1}(a, b)\right)^{1 / p} C_{2}(a, b)$,

$$
C_{3}(a, b) C(Q) \geq e^{\left(\sum \mu_{i} / p-\mu_{n}\right) R}\left(p-\frac{\sum \mu_{i}^{\prime}}{\mu_{n}^{\prime}}\right)^{1 / p} .
$$

We have calculated that $C(Q)=Q^{3+2 / p} e^{(9+3 / p) Q}$. Combining these results and taking the logarithm (note that in the following calculations every constant depending on $\mu$ and $\mu^{\prime}$ can be estimated using $a$ and $b$ ), we get

$$
\left(3+\frac{2}{p}\right) \log Q+\left(9+\frac{3}{p}\right) Q \geq G^{\prime}(a, b)+\left(\frac{\sum \mu_{i}}{p}-\mu_{n}\right) R+\frac{1}{p} \log \left(p-\frac{\sum \mu_{i}^{\prime}}{\mu_{n}^{\prime}}\right)
$$


with some constant $G^{\prime}$ depending only on $a, b . \quad p \geq 1$ hence the left-hand size can be estimated as $5 \log Q+12 Q<24 Q$. Setting $p=\sum \mu_{i}^{\prime} / \mu_{n}^{\prime}+1 / R$, we get

$$
24 Q \geq G^{\prime}(a, b)+\frac{\mu_{n}\left(\frac{\sum \mu_{i}}{\mu_{n}}-\frac{\sum \mu_{i}^{\prime}}{\mu_{n}^{\prime}}-\frac{1}{R}\right) R}{\frac{\sum \mu_{i}^{\prime}}{\mu_{n}^{\prime}}+\frac{1}{R}}+\frac{1}{p} \log \frac{1}{R} .
$$

For $R \geq G^{\prime \prime}(a, b)$ with some well-chosen constant $G^{\prime \prime}$,

$$
24 Q \geq G^{\prime}(a, b)+\frac{\mu_{n} \mu_{n}^{\prime}}{4 \sum \mu_{i}^{\prime}}\left(\frac{\sum \mu_{i}}{\mu_{n}}-\frac{\sum \mu_{i}^{\prime}}{\mu_{n}^{\prime}}\right) R-\frac{\mu_{n}^{\prime}}{2 \sum \mu_{i}^{\prime}} \log R,
$$

and finally we can rewrite our inequality under the desired form

$$
Q \geq G_{1}(a, b)\left(\frac{\sum \mu_{i}}{\mu_{n}}-\frac{\sum \mu_{n}^{\prime}}{\mu_{n}^{\prime}}\right) R-G_{2}(a, b)
$$

with $G_{1}(a, b)$ and $G_{2}(a, b)$ being constants depending only on $a$ and $b$.

This finishes the proof of Theorem 5 .

\section{QUASI-ISOMETRIC Distortion FOR REgUlar TREES}

In this section, we prove that embedding hyperbolic balls into trees requires linear distorsion growth.

First we need coarse notions of volume and of separation (minimal volume of subsets dividing a metric space $X$ into two pieces).

Definition 13. Let $a>0$. We will call the $a$-volume of a metric space $X$ the following quantity

$\operatorname{Vol}_{a}(X)=\sup \left\{v \mid\right.$ for any family $B_{j}$ of balls of radius a covering $\left.X: \#\left\{B_{j}\right\} \geq v\right\}$.

Definition 14. Let $a>0$. We call $a$-separation of $X$ the number

$$
\begin{aligned}
\operatorname{sep}_{a}(X)= & \sup \left\{N \mid \text { for any partition } X=U_{1} \sqcup U_{2} \text { such that } \operatorname{Vol}_{a}\left(U_{i}\right) \geq \operatorname{Vol}_{a}(X) / 3,\right. \\
& i=1,2, \text { for any family } B_{j} \text { of pairwise disjoint balls of radius } a, \\
& \text { \#balls intersecting both } \left.U_{1} \text { and } U_{2} \geq N\right\}
\end{aligned}
$$

Theorem 6. Let $X$ be a bounded metric space, and $T$ be a tree of degree at most $d$. $S=\operatorname{sep}_{a}(X)$ and $V=V_{\operatorname{Vol}_{a}}(X)$. Suppose that for any subset $Y$ of $X$ of a-volume at least one third of $V$, the diameter of $Y$ is at least diam $(X) / D$ for some constant $D$ depending only on $X$. If $f: X \rightarrow T$ is a $(\lambda, c)$-quasi-isometric embedding then

- either $\operatorname{diam}(X) \leq c D$,

- or

$$
\lambda 2 a+c \geq \log _{d} \frac{S}{\operatorname{Vol}_{a}(B(c))} .
$$


Proof. Let $\left\{B_{j}\right\}$ be a maximal set of pairwise disjoint balls of radius $a$. We consider $T$ as a finite discrete metric space. If there exists a vertex $t$ of $T$ such that at least one third of centers of $B_{j}$ are sent to $t$ then $\operatorname{diam}(X) \leq c D$ because of the hypothesis on the space $X$. Otherwise, for any vertex $t$,

$$
\operatorname{Vol}_{a}\left(f^{-1}(t)\right)<\operatorname{Vol}_{a}(X) / 3 .
$$

We are going to find a vertex $t$ which divides the tree into two components $T=T_{1} \cup$ $T_{2}, T_{1} \cap T_{2}=\{t\}$ such that $\operatorname{Vol}_{a} f^{-1}\left(T_{i}\right), i=1,2$, is at least one third of $\operatorname{Vol}_{a}(X)$. To show this, it suffices to start from some boundary vertex (we will call $T_{1}$ the component which contains the initial vertex) of the tree and to pass from one vertex to another. At every step we choose a vertex which increases $\operatorname{Vol}_{a} f^{-1}\left(T_{1}\right)$. We finish when the accumulated volume is sufficient, that is $\operatorname{Vol}_{a} f^{-1}\left(T_{1}\right) \geq \operatorname{Vol}_{a}(X) / 3$.

Denote by $U_{i}=f^{-1}\left(T_{i}\right), i=1,2$. The number $N_{s}$ of balls $B_{j}$ which intersect both $U_{1}$ and $U_{2}$ is at least $N_{s} \geq \operatorname{sep}_{a}(X)=S$. Let $I$ be a set which contains a point of the intersection $U_{1} \cap B_{j}$ for all such balls, denote the image of $I$ by $I^{\prime}=f(I)$.

$$
\left|I^{\prime}\right| \geq \frac{S}{\operatorname{Vol}_{a}(B(c))} .
$$

Because $I^{\prime} \subset T_{1}$, there exists $v_{1} \in I^{\prime}$ such that

$$
d\left(v_{1}, T_{2}\right) \geq \log _{d} \frac{S}{\operatorname{Vol}_{a}(B(c))} .
$$

Thus $v_{1}=f\left(u_{1}\right)$ and $u_{1} \in B_{j}$ which intersects $U_{2}$, there exists $u_{2} \in U_{2} \cap B_{j}$ and $d\left(u_{1}, u_{2}\right) \leq$ $2 a$, hence $d\left(v_{1}, f\left(u_{2}\right)\right) \leq \lambda 2 a+c$. Hence,

$$
\lambda 2 a+c \geq \log _{d} \frac{S}{\operatorname{Vol}_{a}(B(c))} .
$$

Consider $\mathbb{H}^{n}$ with $n \geq 3$. For a ball of radius $R$ in $\mathbb{H}^{n}$ we have $S \sim e^{(n-2) R}$ (we will prove this soon, in Lemma 9), $V \sim e^{(n-1) R}$ and $D=1$. Then the application of Theorem 6 to $B(R) \subset \mathbb{H}^{n}$ with $n \geq 3$ proves the linear quasi-isometric distortion between $\mathbb{H}^{n}$ and a regular tree.

Corollary 1. The quasi-isometric distortion growth for hyperbolic space $\mathbb{H}^{n}, n \geq 3$, and a regular tree is linear in $R$.

Lemma 9. Let $B(R):=B_{\mathbb{H}^{n}}(R)=A_{1} \sqcup A_{2}$ be a partition of an $R$-ball of hyperbolic $n$ space. Suppose that both pieces have large volume: $\operatorname{Vol} A_{i} \geq 1 / 3 \operatorname{Vol} B(R), i=1,2$. Then for $R$ large enough the volume of the common boundary of $A_{1}$ and $A_{2}, S_{12}=\partial A_{1} \cap \partial A_{2}$ is at least

$$
\operatorname{Vol} S_{12} \geq \operatorname{const}(n) e^{(n-2) R},
$$

where the multiplicative constant depends only on dimension $n$. 
Proof. Consider the indicator function $\hat{\iota}$ of $A_{1}$

$$
\hat{\imath}(x)= \begin{cases}1 & x \in A_{1} \\ 0 & x \in A_{2}\end{cases}
$$

We would like to write 1-Poincaré inequality for $\hat{\imath}$ but first we have to make it smooth.

Fix two real numbers $r_{1}<r_{2}$. Take an $r_{1}$-separated and $r_{2}$-dense set $S$. For any point $z \in S$ we define a function $h_{z}$ as follows,

$$
\hat{h}_{z}(x)=\left\{\begin{array}{cl}
1-\frac{d(x, z)}{2 R} & x \in B\left(z, 2 r_{2}\right) \\
0 & x \in B\left(z, 2 r_{2}\right)
\end{array} .\right.
$$

Now set $h_{z}(x)=\hat{h}_{z}(x) / \sum \hat{h}_{z^{\prime}}(x)$ which gives a partition of unity. Evidently gradients of $h_{z}$ are uniformly bounded in function of $r_{1}, r_{2}$ and a number $L\left(r_{1}, r_{2}, n\right)$ of disjoint balls of radius $r_{1}$ in a ball of radius $2 r_{2}: \nabla h_{z} \leq N\left(r_{1}, r_{2}, n\right)$. Now we set

$$
\iota(x)=\sum_{z \in S} \iota(z) h_{z}(x) .
$$

Then

$$
\nabla \iota(x)=\sum_{z \in S} \iota(z) \nabla h_{z}(x) \leq L\left(r_{1}, r_{2}, n\right) N\left(r_{1}, r_{2}, n\right)
$$

because $\iota$ takes only two values 0 and 1 and for any $x$ there is not more than $L\left(r_{1}, r_{2}, n\right)$ functions $h_{z}$ which do not vanish at $x$. Now we notice that outside of $2 r_{2}$-tubular neighbourhood $T_{12}$ of $S_{12}=\partial A_{1} \cap \partial A_{2}, \iota$ coincides with $\hat{\iota}$. We notice here that $\operatorname{VolT}_{12} \sim \operatorname{Vol} S_{12}$ (up to some multiplicative constants depending on $n$ and $\left.r_{2}\right)$. If $\operatorname{Vol}\left(A_{1} \backslash T_{12}\right)$ or $\operatorname{Vol}\left(A_{2} \backslash T_{12}\right)$ is too small, then there is nothing to prove as $\operatorname{VolT}_{12} \sim \operatorname{Vol} B(R)$. Otherwise we write Poincaré inequality for $\iota$.

The mean value $\operatorname{Vol}\left(A_{1} \backslash T_{12}\right) \leq \oint \iota d \operatorname{Vol} \leq \operatorname{Vol}\left(A_{1} \backslash T_{12}\right)+\left.\sup \nabla \iota\right|_{T_{12}} \operatorname{Vol} T_{12}$, that is

$$
c_{\iota}=\oint \iota d V o l \sim \operatorname{Vol} A_{1} .
$$

We are ready to write 1-Poincaré inequality for $\iota$ for $R$ large enough,

$$
\int\left|\iota-c_{\iota}\right| d V o l \leq \operatorname{const}(n) e^{R} \int|\nabla \iota| d V o l,
$$

where $\operatorname{const}(n)$ is some constant depending only on dimension $n$. We compute the left-hand integral,

$$
\int\left|\iota-c_{\iota}\right| d V o l \approx \int_{A_{1}}\left|1-c_{\iota}\right| d V o l+\int_{A_{2}} c_{\iota} d V o l=\left(1-c_{\iota}\right) \operatorname{Vol} A_{1}+c_{\iota} \operatorname{Vol} A_{2} \geq \frac{2}{3} \operatorname{Vol} B(R) .
$$

For the right-hand integral, we obtain

$$
\int|\nabla \iota| d V o l=\int_{T_{12}}|\nabla \iota| d V o l \leq \sup \nabla \iota V o l T_{12}=\operatorname{const}(n) \operatorname{Vol} S_{12} .
$$

Combining all these inequalities we conclude that

$$
\operatorname{VolB}(R) \leq \operatorname{const}(n) e^{R} \operatorname{Vol} S_{12} \text {. }
$$


As for $R$ large enough $\operatorname{Vol} B(R) \sim e^{(n-1) R}$ we finish the proof with the needed result

$$
\operatorname{Vol} S_{12} \geq \operatorname{const}(n) e^{(n-2) R}
$$

Question 1. What is the quasi-isometric distortion between a d-regular tree and hyperbolic plane $\mathbb{H}^{2}$.

\section{Approximation of Distances AND RADial QUASI-ISOMETRIES}

9.1. Orthogonal triangles in hyperbolic spaces. At the beginning of this section we give to lemmas on the geometry of orthogonal triangles in hyperbolic spaces. The second Lemma will be used to establish an approximation of distances in hyperbolic spaces which allow to control a quasi-isometric action.

Lemma 10. Let $\sigma$ be a geodesic segment, a be a point not on $\sigma$, and $c$ be a projection of $a$ on $\sigma$. Let $b \in \sigma$ be arbitrary, and let d denote a projection of $b$ on ac. Then $|c-d| \leq 2 \delta$.

Proof. By hypothesis, $b d$ minimizes the distance of $b$ to any point of $a c$, and because the triangle $b c d$ is $\delta$-thin, there exists a point $e \in b d$ such that $d(e, a c)=|e-d| \leq \delta$ and $d(e, b c) \leq \delta$. Because $a c$ is a perpendicular to $\sigma,|a-c| \leq|a-d|+|d-e|+d(e, b c) \leq|a-d|+2 \delta$. Hence $|c-d| \leq 2 \delta$.

Lemma 11. As in the preceding lemma, let $\sigma$ be a geodesic segment, a be a point not on $\sigma, c$ be a projection of $a$ on $\sigma$, and $b$ be some point on $\sigma$. Let d denote a point on ac such that $|d-c|=\delta$ and $e$ denote a point on bc such that $|e-c|=3 \delta$. Then

- $d(d, a b) \leq \delta, d(e, a b) \leq \delta, d(c, a b) \leq 2 \delta$, and

- the length of ab differs from the sum of the lengths of the two other sides by at most $8 \delta$,

$$
|a-c|+|b-c|-2 \delta \leq|a-b| \leq|a-c|+|b-c|+8 \delta .
$$

Proof. The triangle $a b c$ is $\delta$-thin. Therefore, obviously, $d(d, a b) \leq \delta$ (the distance from a point of $a c$ to $a b$ is a continuous function). We take a point $x \in b c$ such that $d(x, c a) \leq \delta$. Using Lemma 10, we obtain $|b-x|+d(x, c a) \geq|b-c|-2 \delta$, and hence $|c-x| \leq d(x, c a)+2 \delta \leq$ $3 \delta$.

We now let $d_{1}$ and $e_{1}$ denote respective projections of $d$ and $e$ on $a b$. Then by the triangle inequality, we have

- $|a-d|-\delta \leq\left|a-d_{1}\right| \leq|a-d|+\delta$,

- $|b-e|-\delta \leq\left|b-e_{1}\right| \leq|b-e|+\delta$, and

- $0 \leq\left|d_{1}-e_{1}\right| \leq\left|d_{1}-d\right|+|d-c|+|c-e|+\left|e-e_{1}\right| \leq 6 \delta$.

Combining all these inequalities, we obtain the second point in the lemma. 
9.2. Approximation of distances in hyperbolic metric spaces. Let $X, Y$ be two geodesic hyperbolic metric spaces with base points $x_{0} \in X, y_{0} \in Y$. Let $\theta: \partial X \rightarrow \partial Y$ be a homeomorphism between ideal boundaries.

Hypothesis 1 . Assume that there exists a constant $D$ such that for any $x \in X$ there exists a geodesic ray $\gamma$ from the base point $\gamma(0)=x_{0}$ and passing near $x: d(x, \gamma)<D$.

We are going to construct approximately (up to $D$ ) a map $\Theta: X \rightarrow Y$ extending the boundary homeomorphism $\theta$. Take some point $x$ and a geodesic ray $\gamma$ from $x_{0}$ passing near $x: d(\gamma, x)<D$. Then $\gamma(\infty)$ is a point on ideal boundary $\partial X$. The corresponding point $\theta(\gamma(\infty)) \in \partial Y$ defines a geodesic ray $\gamma^{\prime}$ such that $\gamma^{\prime}(0)=y_{0}$ and $\gamma^{\prime}(\infty)=\theta(\gamma(\infty))$. Set $\Theta(x)=\gamma^{\prime}\left(d\left(x_{0}, x\right)\right)$. So, by construction, $\Theta$ preserves the distance to the base point. Still, it depends on the choices of $\gamma$ and $\gamma^{\prime}$.

Definition 15. Define the following quantity

$$
K(R)=\sup \left\{\left|\log \frac{d_{y_{0}}\left(\theta\left(\xi_{1}\right), \theta\left(\xi_{2}\right)\right)}{d_{x_{0}}\left(\xi_{1}, \xi_{2}\right)}\right| \mid d_{x_{0}}\left(\xi_{1}, \xi_{2}\right) \geq e^{-R} \vee d_{y_{0}}\left(\theta\left(\xi_{1}\right), \theta\left(\xi_{2}\right)\right) \geq e^{-R}\right\} .
$$

We are going to prove that the restriction of $\Theta$ on the ball $B(R) \subset X$ of radius $R$ is a $\left(1+2 \frac{K(R)}{D+\delta}, D+\delta+2 K(R)\right)$-quasi-isometry. We begin with a Lemma which gives an approximation (up to an additive constant) of the distance between two points in a hyperbolic metric space. In its proof, all equalities hold with a bounded additive error depending linearly on $\delta$.

Lemma 12. Let $P_{1}, P_{2}$ be two points in a hyperbolic metric space $Z$. Let $P_{0}$ be a base point (possibly at infinity). Let distances (horo-distances if $P_{0}$ is at infinity) from $P_{1}$ and $P_{2}$ to $P_{0}$ be $d\left(P_{1}, P_{0}\right)=t_{1}$ and $d\left(P_{2}, P_{0}\right)=t_{2}$. Assume that there exist points $P_{1}^{\infty}$ and $P_{2}^{\infty}$ such that $P_{1}$ (resp. $P_{2}$ ) belongs to the geodesic ray defined by $P_{0}$ and $P_{1}^{\infty}$ (resp. $\left.P_{2}^{\infty}\right)$. Denote $b y^{11}$

$$
t_{\infty}=-\log \text { visdist }_{P_{0}}\left(P_{1}^{\infty}, P_{2}^{\infty}\right)
$$

the logarithm of visual distance seen from $P_{0}$. Then up to adding a multiple of $\delta$,

$$
d\left(P_{1}, P_{2}\right)=t_{1}+t_{2}-2 \min \left\{t_{1}, t_{2}, t_{\infty}\right\} .
$$

Proof. Let $P_{0}^{\prime}$ be a projection of $P_{0}$ on the geodesic $P_{1}^{\infty} P_{2}^{\infty}$. By Lemma 11, $P_{0}^{\prime}$ lies at distance at most $2 \delta$ from both $P_{0} P_{1}^{\infty}$ and $P_{0} P_{2}^{\infty}$. Hence, up to an additive constant bounded by $4 \delta$ the distance between $P_{0}$ and $P_{0}^{\prime}$ is equal to Gromov's product of $P_{1}^{\infty}$ and $P_{2}^{\infty}$. It follows that $t_{\infty}=d\left(P_{0}, P_{0}^{\prime}\right)=-\log \operatorname{visdist}\left(P_{1}, P_{2}\right)$.

The triangle $P_{0} P_{1}^{\infty} P_{2}^{\infty}$ is $\delta$-thin. Notice that if $P_{1}$ (or $P_{2}$ ) lies near the side $P_{1}^{\infty} P_{2}^{\infty}$ then $t_{1} \geq t_{\infty}$. Otherwise, $t_{1} \leq t_{\infty}$ (both inequalities are understood up to an additive error $\delta$ ). This follows from the definition of the point $P_{0}^{\prime}$ as a projection and Lemma 11.

\footnotetext{
${ }^{1}$ We define visdist $\left(P_{1}^{\infty}, P_{2}^{\infty}\right)$ of two points $P_{1}^{\infty}, P_{2}^{\infty}$ at the ideal boundary as the exponential of minus Gromov's product of these points $e^{-\left(P_{1}^{\infty} \mid P_{2}^{\infty}\right)}$. Indeed, it is not a distance as it does not satisfy triangle inequality. But we will never have more than two points at infinity at the same time in our setting, so we will not use this property.
} 
Hence, if $t_{1}, t_{2} \geq t_{\infty}, d\left(P_{1}, P_{2}\right)=d\left(P_{1}, P_{0}\right)+d\left(P_{2}, P_{0}\right)-2 d\left(P_{0}, P_{0}^{\prime}\right)=t_{1}+t_{2}-2 t_{\infty}$. If $t_{1} \leq t_{\infty} \leq t_{2}, d\left(P_{1}, P_{2}\right)=d\left(P_{1}, P_{0}^{\prime}\right)+d\left(P_{0}^{\prime}, P_{2}\right)=t_{2}-t_{1}$.

Finally, if $t_{1}, t_{2} \leq t_{\infty}$, we get $d\left(P_{1}, P_{2}\right)=\left|t_{1}-t_{2}\right|=t_{1}+t_{2}-2 \min \left\{t_{1}, t_{2}\right\}$ as $P_{1}$ lies near $P_{0} P_{2}^{\infty}$.

9.3. Construction of quasi-isometry. Although the quasi-isometry which will be constructed in this section can seem to be a bit naive, it will allow us to establish an example of logarithmic quasi-isometric distortion in section 10.2

Lemma 13. Let $Z$ and $Z^{\prime}$ be two hyperbolic metric spaces. Let $\Theta$ be the radial extension of a boundary homeomorphism $\theta$, as described at the beginning of this section. Then for any two points $P_{1}, P_{2} \in B\left(P_{0}, R\right) \subset Z$ such that $d\left(P_{1}, P_{2}\right)>c$, we have

$$
\frac{d_{Z^{\prime}}\left(\Theta\left(P_{1}\right), \Theta\left(P_{2}\right)\right)}{d_{Z}\left(P_{1}, P_{2}\right)} \leq 1+2 \frac{K(R)}{c} .
$$

If $d\left(P_{1}, P_{2}\right)<c$,

$$
d_{Z^{\prime}}\left(\Theta\left(P_{1}\right), \Theta\left(P_{2}\right)\right)<2 K(R)+c .
$$

Proof. We will use the same notations as in Lemma12. Visual distance $d_{Z}^{\infty}$ between $P_{1}^{\infty}$ and $P_{2}^{\infty}$ and the (horo-)distance $t_{\infty}$ from $P_{0}$ to $P_{1}^{\infty} P_{2}^{\infty}$ are connected by the relation $e^{-t_{\infty}}=$ $d_{\infty}\left(P_{1}^{\infty}, P_{2}^{\infty}\right)$. In the same way we define $t_{\infty}^{\prime}$ as the (horo-)distance for corresponding images.

By Lemma 12 we know that $d\left(P_{1}, P_{2}\right)=t_{1}+t_{2}-2 \min \left\{t_{1}, t_{2}, t_{\infty}\right\}$.

Assume first $d\left(P_{1}, P_{2}\right)>c$. We will write $d_{Z}=d\left(P_{1}, P_{2}\right)$ for the distance between $P_{1}$ and $P_{2}$ and $d_{Z^{\prime}}=d\left(\Theta\left(P_{1}\right), \Theta\left(P_{2}\right)\right)$ for the distance between their images.

We have to consider four cases depending on the relative sizes of $t_{1}, t_{2}, t_{0}$ and $t_{\infty}^{\prime}$ as they determine values of minima defining $d_{Z}$ and $d_{Z^{\prime}}$. Without loss of generality, we may assume that $t_{1} \leq t_{2}$.

1 st case. If both $t_{1}<t_{\infty}$ and $t_{1}<t_{\infty}^{\prime}$, then

$$
\frac{d_{Z^{\prime}}}{d_{Z}}=\frac{t_{2}-t_{1}}{t_{2}-t_{1}}=1
$$

and this case is trivial.

2nd case. If $t_{\infty}<t_{1}$ and $t_{\infty}^{\prime}<t_{1}$. We have to give an upper bound for

$$
\frac{d_{Z^{\prime}}}{d_{Z}}=\frac{t_{1}+t_{2}-2 t_{\infty}^{\prime}}{t_{1}+t_{2}-2 t_{0}^{\infty}}
$$

Consider

$$
t_{\infty}^{\prime}-t_{\infty}=\log \frac{d_{\infty}\left(\theta\left(P_{1}^{\infty}\right), \theta\left(P_{2}^{\infty}\right)\right)}{d_{\infty}\left(P_{1}^{\infty}, P_{2}^{\infty}\right)}
$$

Because $d_{Z}>c$, we have $t_{1}+t_{2}-2 t_{\infty}>c$ hence $e^{\left(t_{1}+t_{2}\right) / 2} e^{-t_{\infty}}>e^{c / 2}$. And as $t_{1}, t_{2} \leq R$ we obtain for visual distance $d_{Z}^{\infty} \geq e^{c / 2} e^{-R} \geq e^{-R}$. We conclude that

$$
\left|t_{\infty}^{\prime}-t_{\infty}\right| \leq K(R)
$$


Finally,

$$
\frac{d_{Z^{\prime}}}{d_{Z}}=\frac{d_{Z^{\prime}}-d_{Z}+d_{Z}}{d_{Z}}=1+\frac{t_{\infty}^{\prime}-t_{\infty}}{t_{1}+t_{2}-t_{\infty}} \leq 1+\frac{1}{c}\left|t_{\infty}^{\prime}-t_{\infty}\right| .
$$

$3 d$ case. Now let $t_{\infty}<t_{1}<t_{\infty}^{\prime}$. Then

$$
d_{Z^{\prime}}-d_{Z}=t_{2}-t_{1}-\left(t_{1}+t_{2}-2 t_{\infty}\right)=2\left(t_{\infty}-t_{1}\right) \leq 0,
$$

which leads to

$$
\frac{d_{Z^{\prime}}}{d_{Z}} \leq 1
$$

4th case. Finally if $t_{\infty}^{\prime}<t_{1}<t_{0}^{\infty}$ then

$$
d_{Z^{\prime}}-d_{Z}=\left(t_{1}+t_{2}-2 t_{\infty}^{\prime}\right)-\left(t_{2}-t_{1}\right)=2\left(t_{1}-t_{\infty}^{\prime}\right) \leq 2\left(t_{0}^{\infty}-t_{\infty}^{\prime}\right) .
$$

We know that $t_{1} \leq R$ and at the same time we have $t_{\infty}^{\prime}<t_{1}$, hence $t_{\infty}^{\prime}<R$ and visual distance between $P_{1}^{\infty \prime}$ and $P_{2}^{\infty \prime}$ is at least $e^{-R}$. Now as in the 2 nd case we obtain that $t_{0}^{\infty}-t_{\infty}^{\prime} \leq K(R)$ and hence

$$
\frac{d_{Z^{\prime}}}{d_{Z}} \leq 1+2 \frac{K(R)}{c}
$$

Now assume that $d_{Z}\left(P_{1}, P_{2}\right) \leq c$ (we still suppose $\left.t_{1} \leq t_{2}\right)$, hence the distance $t_{\infty}>t_{2}$ and we are either in first or fourth situation. In the first case, $t_{1}<t_{\infty}$ and $t_{1}<t_{\infty}^{\prime}$ so $d_{Z^{\prime}}=$ $d_{Z} \leq c$. In the fourth case, we have still $d_{Z^{\prime}}-d_{Z} \leq 2 K(R)$ and hence $d_{Z}^{\prime} \leq c+2 K(R)$.

Applying the Lemma both to $\Theta$ and $\Theta^{-1}$, we get the following Theorem.

Theorem 7. Let $X, Y$ be two geodesic hyperbolic metric spaces with base points $x_{0} \in X$, $y_{0} \in Y$. Assume that there exists a constant $D$ such that for any $x \in X$ there exists a geodesic ray $\gamma$ from the base point $\gamma(0)=x_{0}$ and passing near $x: d(x, \gamma)<D$ (Hypothesis 1). Let the restriction of $\Theta: \partial X \rightarrow \partial Y$ be a homeomorphism between ideal boundaries. Then the restriction of $\Theta$ on a ball $B\left(x_{0}, R\right) \subset X$ of radius $R$ is a $\left(\lambda, C_{q}\right)$-quasi-isometry to $B\left(y_{0}, R\right) \subset Y$, where $\lambda=1+2 \frac{K(R)}{c}$ and $C_{q}=2 K(R)+c$. The constant $c$ can be chosen as $c=D+\delta$ where $\delta$ is the hyperbolicity constant.

\section{EXAmples}

10.1. Bi-Hölder maps. Let $\theta$ be a bi-Hölder map:

$$
\begin{aligned}
& d\left(\theta\left(\xi_{1}\right), \theta\left(\xi_{2}\right)\right) \leq c d\left(\xi_{1}, \xi_{2}\right)^{\alpha}, \alpha<1, \\
& d\left(\theta\left(\xi_{1}\right), \theta\left(\xi_{2}\right)\right) \geq \frac{1}{c} d\left(\xi_{1}, \xi_{2}\right)^{\beta}, \beta>1 .
\end{aligned}
$$

Assume first that for two points $\xi_{1}, \xi_{2}$ of the ideal boundary, the visual distance $d\left(\xi_{1}, \xi_{2}\right)>$ $e^{-R}$. Then we have

$$
\log \frac{d\left(\theta\left(\xi_{1}\right), \theta\left(\xi_{2}\right)\right)}{d\left(\xi_{1}, \xi_{2}\right)} \leq \log c d\left(\xi_{1}, \xi_{2}\right)^{\alpha-1}=-(1-\alpha) \log d\left(\xi_{1}, \xi_{2}\right) \lesssim(1-\alpha) R .
$$


Now, if the visual distance between images of $\xi_{1}$ and $\xi_{2}$ satisfy $d\left(\theta\left(\xi_{1}\right), \theta\left(\xi_{2}\right)\right)>e^{-R}$, we get

$$
d\left(\xi_{1}, \xi_{2}\right) \geq \frac{1}{c^{1 / \alpha}} e^{-R / \alpha}
$$

and hence

$$
\log \frac{d\left(\theta\left(\xi_{1}\right), \theta\left(\xi_{2}\right)\right)}{d\left(\xi_{1}, \xi_{2}\right)} \gtrsim \frac{1-\alpha}{\alpha} R .
$$

We obtain the lower bound for $\log \frac{d\left(\theta\left(\xi_{1}\right), \theta\left(\xi_{2}\right)\right)}{d\left(\xi_{1}, \xi_{2}\right)}$ just in the same way as the upper-bound. If $d\left(\xi_{1}, \xi_{2}\right)>e^{-R}$

$$
\log \frac{d\left(\theta\left(\xi_{1}\right), \theta\left(\xi_{2}\right)\right)}{d\left(\xi_{1}, \xi_{2}\right)} \geq \log \frac{1}{c} d\left(\xi_{1}, \xi_{2}\right)^{\beta-1}=-(1-\beta) \log d\left(\xi_{1}, \xi_{2}\right) \lesssim(1-\beta) R .
$$

If $d\left(\theta\left(\xi_{1}\right), \theta\left(\xi_{2}\right)\right)>e^{-R}$

$$
\log \frac{d\left(\theta\left(\xi_{1}\right), \theta\left(\xi_{2}\right)\right)}{d\left(\xi_{1}, \xi_{2}\right)} \geq \log \frac{1}{c} d\left(\theta\left(\xi_{1}\right), \theta\left(\xi_{2}\right)\right)^{(\beta-1) / \beta}=-\frac{1-\beta}{\beta} \log d\left(\theta\left(\xi_{1}\right), \theta\left(\xi_{2}\right)\right) \gtrsim \frac{1-\beta}{\beta} R .
$$

This gives

$$
K(R) \lesssim \max \{1-\alpha, 1-\beta\} R .
$$

In particular, consider two variants of the space $T^{n} \times[0,+\infty) Z$ and $Z^{\prime}$ with metrics $d t^{2}+\sum e^{2 \mu_{i} t} d x_{i}^{2}$ and $d t^{2}+\sum e^{2 \mu_{i}^{\prime} t} d x_{i}^{2}$ respectively. The visual distance between points $P_{1}$ and $P_{2}$ is given by

$$
d_{\infty}\left(P_{1}, P_{2}\right) \sim \max \left|x_{i}^{1}-x_{i}^{2}\right|^{1 / \mu_{i}}
$$

Pick the identity map $\theta: \partial Z \rightarrow \partial Z^{\prime}$. Then

$$
\frac{d_{\infty}\left(\theta\left(P_{1}\right), \theta\left(P_{2}\right)\right)}{d_{\infty}\left(P_{1}, P_{2}\right)} \sim \frac{\max _{i}\left|x_{i}^{1}-x_{i}^{2}\right|^{1 / \mu_{i}^{\prime}}}{\max _{i}\left|x_{i}^{1}-x_{i}^{2}\right|^{1 / \mu_{i}}} \leq \max _{i}\left|x_{i}^{1}-x_{i}^{2}\right|^{1 / \mu_{i}^{\prime}-1 / \mu_{i}}
$$

Suppose that $d\left(P_{1}, P_{2}\right)>e^{-R}$. Then

$$
\begin{aligned}
& \left|\log \frac{d_{\infty}\left(\theta\left(P_{1}\right), \theta\left(P_{2}\right)\right)}{d_{\infty}\left(P_{1}, P_{2}\right)}\right| \leq\left|\log \max _{i}\right| x_{i}^{1}-\left.x_{i}^{2}\right|^{1 / \mu_{i}^{\prime}-1 / \mu_{i}} \mid= \\
= & \max _{i}\left(\mu_{i}\left|\frac{1}{\mu_{i}^{\prime}}-\frac{1}{\mu_{i}}\right||\log | x_{i}^{1}-\left.x_{i}^{2}\right|^{1 / \mu_{i}} \mid\right) \leq \max _{i}\left|\frac{\mu_{i}}{\mu_{i}^{\prime}}-1\right| R .
\end{aligned}
$$

So, we conclude that $K(R)=\left|\max _{i}\left(\mu_{i} / \mu_{i}^{\prime}\right)-1\right| R$.

Remark 3. More generally, such bi-Hölder maps exist between boundaries of arbitrary simply connected Riemannian manifolds with bounded negative sectional curvature. The Hölder exponent is controlled by sectional curvature bounds. 
10.2. Unipotent locally homogeneous space. Now assume the space $Z$ is a quotient $\mathbb{R}^{2} / \mathbb{Z}^{2} \times \mathbb{R}$ of the space $\mathbb{R}^{2} \times \mathbb{R}$ with the metric $d t^{2}+e^{2 t}\left(d x^{2}+d y^{2}\right)$. Consider the space $Z^{\prime}=\mathbb{R}^{2} / \mathbb{Z}^{2} \ltimes_{\alpha} \mathbb{R}$, quotient of the space $\mathbb{R}^{2} \rtimes_{\alpha} \mathbb{R}$, where $\alpha$ is the $2 \times 2$ matrix

$$
\left(\begin{array}{ll}
1 & 1 \\
0 & 1
\end{array}\right)
$$

The locally homogeneous metric is of the form $d t^{2}+g_{t}$ where $g_{t}=\left(e^{t \alpha}\right)^{*} g_{0}$

$$
e^{t \alpha}\left(\begin{array}{l}
x \\
y
\end{array}\right)=\left(\begin{array}{cc}
e^{t} & t e^{t} \\
0 & e^{t}
\end{array}\right)\left(\begin{array}{l}
x \\
y
\end{array}\right)=\left(\begin{array}{c}
e^{t} x+t e^{t} y \\
e^{t} y
\end{array}\right)
$$

and so $g_{t}=d\left(e^{t} x+t e^{t} y\right)^{2}+d\left(e^{t} y\right)^{2}=e^{2 t}\left(d x^{2}+2 t d x d y+\left(t^{2}+1\right) d y^{2}\right)$.

Let $\theta: \partial Z \rightarrow \partial Z^{\prime}$ be the identity. Consider two points $P_{1}=\left(x_{1}, y_{1}\right)$ and $P_{2}=\left(x_{2}, y_{2}\right)$ in $Z$. We will write $x=x_{1}-x_{2}$ and $y=y_{1}-y_{2}$. For the visual distance between $P_{1}, P_{2}$ we have

$$
d_{\infty}\left(P_{1}, P_{2}\right)=\max \{|x|,|y|\} .
$$

For their images $\theta\left(P_{1}\right)$ and $\theta\left(P_{2}\right)$ (see section 5 of [4] and [5])

$$
d_{\infty}\left(\theta\left(P_{1}\right), \theta\left(P_{2}\right)\right)=\max \{|y|,|x-y \log | y \mid\} .
$$

First we will give an upper-bound for $\log \left(d_{\infty}\left(\theta\left(P_{1}\right), \theta\left(P_{2}\right)\right) / d_{\infty}\left(P_{1}, P_{2}\right)\right)$. We have four different cases.

1st case. If $|x|<|y|$ and $|x-y \log | y||<|y|$,

$$
\frac{d_{\infty}\left(\theta\left(P_{1}\right), \theta\left(P_{2}\right)\right)}{d_{\infty}\left(P_{1}, P_{2}\right)}=1
$$

2nd case. If $|x-y \log | y||<|y|<|x|$,

$$
\frac{d_{\infty}\left(\theta\left(P_{1}\right), \theta\left(P_{2}\right)\right)}{d_{\infty}\left(P_{1}, P_{2}\right)}<1 .
$$

3d case. If $|x|<|y|<|x-y \log | y||$.

$$
\frac{d_{\infty}\left(\theta\left(P_{1}\right), \theta\left(P_{2}\right)\right)}{d_{\infty}\left(P_{1}, P_{2}\right)}=\frac{|x-y \log y|}{|y|} \leq \frac{|x|}{|y|}+|\log | y|| .
$$

If $d_{\infty}\left(P_{1}, P_{2}\right)>e^{-R}$ we have $e^{-R}<|y| \leq 1$ (the upper bound follows from the fact that $y$ is a coordinate of a point of a torus) and hence $|\log | y|| \leq R$ and we finish as follows,

$$
\frac{d_{\infty}\left(\theta\left(P_{1}\right), \theta\left(P_{2}\right)\right)}{d_{\infty}\left(P_{1}, P_{2}\right)} \leq \frac{|x|}{|y|}+|\log | y|| \leq 1+R
$$

If $d_{\infty}\left(\theta\left(P_{1}\right), \theta\left(P_{2}\right)\right)>e^{-R}$ we will consider two situations.

- If $|x|>|y \log | y||$ then $|x-y \log y|<2|x|$ and as $|x|<|y|$,

$$
\frac{d_{\infty}\left(\theta\left(P_{1}\right), \theta\left(P_{2}\right)\right)}{d_{\infty}\left(P_{1}, P_{2}\right)} \leq 2
$$


- If $|x|<|y \log | y||$ then $e^{-R}<|x-y \log | y||<2|y \log | y||$ and hence $|\log | y||<R$, so

$$
\frac{d_{\infty}\left(\theta\left(P_{1}\right), \theta\left(P_{2}\right)\right)}{d_{\infty}\left(P_{1}, P_{2}\right)} \leq 1+R
$$

4th case. Let now $|y|<|x|$ and $|y|<|x-y \log | y||$

$$
\frac{d_{\infty}\left(\theta\left(P_{1}\right), \theta\left(P_{2}\right)\right)}{d_{\infty}\left(P_{1}, P_{2}\right)}=\frac{|x-y \log | y||}{|x|} \leq 1+\frac{|y \log | y||}{|x|} .
$$

We will check two possibilities.

- If $|y| \leq|x|^{2}$ then

$$
\frac{|y \log | y||}{|x|}=\left.\frac{|y|^{1 / 2}}{|x|}|| y\right|^{1 / 2} \log |y| \mid \leq 1 .
$$

- Now suppose that $|y| \geq|x|^{2}$. If $d_{\infty}\left(P_{1}, P_{2}\right)>e^{-R}$, we see easily that $|y| \geq e^{-2 R}$ and hence

$$
\frac{|y \log | y||}{|x|} \leq \frac{|x \log | y||}{|x|} \leq|\log | y|| \leq 2 R \text {. }
$$

If $d_{\infty}\left(\theta\left(P_{1}\right), \theta\left(P_{2}\right)\right)>e^{-R}$ we use the fact that $|a+b| \geq 2 \max \{|a|,|b|\}$. Hence, either $|x|>e^{-R} / 2$ or $|y \log | y||>e^{-R} / 2$ and so $|y| \gtrsim e^{-R}$ and we finish the estimation as earlier.

So in the fourth case we have also

$$
\frac{d_{\infty}\left(\theta\left(P_{1}\right), \theta\left(P_{2}\right)\right)}{d_{\infty}\left(P_{1}, P_{2}\right)} \leq 2 R
$$

Here, we have proved that $\log \left(d_{\infty}\left(\theta\left(P_{1}\right), \theta\left(P_{2}\right)\right) / d_{\infty}\left(P_{1}, P_{2}\right)\right) \leq \log R$. Now we proceed to give also a lower bound for this expression.

1st case. If $|x|<|y|$ and $|x-y \log | y||<|y|$,

$$
\frac{d_{\infty}\left(\theta\left(P_{1}\right), \theta\left(P_{2}\right)\right)}{d_{\infty}\left(P_{1}, P_{2}\right)}=1
$$

2nd case. If $|x-y \log | y||<|y|<|x|$,

$$
\frac{d_{\infty}\left(\theta\left(P_{1}\right), \theta\left(P_{2}\right)\right)}{d_{\infty}\left(P_{1}, P_{2}\right)}=\frac{|y|}{|x|} .
$$

Without loss of generality, assume $x>0$. By the construction of $Z,|y|<1$ hence $\log |y|<$ 0 . If $0<x \leq y \log |y|$, we have $y<0$. Now transform $x \leq y \log |y|$ as $1 \leq-\log |y|(-y) / x$, hence

$$
-\frac{y}{x} \geq-\frac{1}{\log |y|}
$$

Now either $d_{\infty}\left(\theta\left(P_{1}\right), \theta\left(P_{2}\right)\right)=|y|>e^{-R}$ or $e^{-R} \leq d_{\infty}\left(P_{1}, P_{2}\right)=|x| \leq y \log |y|$ which also means that $|y| \gtrsim e^{-R}$. So,

$$
\frac{|y|}{|x|} \geq \frac{1}{R}
$$


If on the contrary $y \log |y| \leq x$ we have

$$
x-y \log |y|<|y|<x .
$$

First we notice that $y \log |y|>x-|y|>0$. As $|y|<1$ for any point of our space, $\log |y|<0$ and we conclude that $y<0$. Now from ([6) we obtain that $x<-y(1-\log |y|)$. As $1-\log |y|>0$ we obtain

$$
-\frac{y}{x}>\frac{1}{1-\log |y|}
$$

If $d_{\infty}\left(\theta\left(P_{1}\right), \theta\left(P_{2}\right)\right)=|y|>e^{-R}$, we trivially get that

$$
\frac{|y|}{|x|}>\frac{1}{R}
$$

If $e^{-R} \leq d_{\infty}\left(P_{1}, P_{2}\right)=|x|$ we write $e^{-R}<x<-y(1-\log |y|)$ and hence $y \gtrsim e^{-R}$, so we obtain the same result. So, in both cases we come to the same result

$$
\left|\log \frac{|y|}{|x|}\right|<R \text {. }
$$

$3 d$ case. Assume $|x|<|y|<|x-y \log | y||$, this case is trivial as

$$
\frac{d_{\infty}\left(\theta\left(P_{1}\right), \theta\left(P_{2}\right)\right)}{d_{\infty}\left(P_{1}, P_{2}\right)}=\frac{|x-y \log y|}{|y|} \geq 1 .
$$

4th case. Let now $|y|<|x|$ and $|y|<|x-y \log | y||$. We also suppose that $x>0$ to save notation.

$$
\frac{d_{\infty}\left(\theta\left(P_{1}\right), \theta\left(P_{2}\right)\right)}{d_{\infty}\left(P_{1}, P_{2}\right)}=\frac{|x-y \log | y||}{|x|}=\left|1-\frac{y \log |y|}{x}\right| .
$$

If (77) is greater than $1 / 2$ then we have nothing to prove. So suppose that (7) is less than $1 / 2$

$$
-\frac{x}{2} \leq x-y \log |y| \leq \frac{x}{2}
$$

and so

$$
\frac{x}{2} \leq y \log |y| \leq \frac{3 x}{2}
$$

The last inequality shows that if either $d_{\infty}\left(\theta\left(P_{1}\right), \theta\left(P_{2}\right)\right) \geq e^{-R}$ or $d_{\infty}\left(P_{1}, P_{2}\right) \geq e^{-R}$, $|y| \gtrsim e^{-R}$ and so we have

$$
\frac{|y \log | y||}{x} \geq \frac{|y \log | y||}{y}=|\log | y|| \geq \frac{1}{R}
$$

which completes our discussion of this example. We have proved that

$$
K(R) \lesssim \log R
$$




\section{Appendix: Quasi-isometric embeddings and Fundamental groups}

Here we would like to discuss the hypothesis of the Theorem 5 that the quasi-isometric embedding under consideration is a homotopy equivalence. We will show that if $\operatorname{dim}(Z) \geq$ 3 , one may believe that the assumption that $\Theta$ be isomorphic on fundamental groups is not that restrictive. Indeed, in Proposition 6, we shall show that this is automatic, but unfortunately the argument introduces an ineffective constant $R_{0}$, which makes it useless. For instance, if it turns out that $R_{0}=\lambda_{1}^{2}$, Proposition [6 does not help to remove the homotopy assumption in Theorem 5. Nevertheless, it is included for completeness sake.

Proposition 6. Let $Z, Z^{\prime}$ be two spaces of the described form with equal dimensions $n+1 \geq$ 3. Then for any $\lambda_{1} \geq 1, \lambda_{2} \geq 1, c_{1} \geq 0, c_{2} \geq 0$ there exists $R_{0}=R_{0}\left(\lambda_{1}, \lambda_{2}, c_{1}, c_{2}\right)$ such that if $R>R_{0}$ and a continuous map $f: B_{Z_{\mu}}\left(O, R_{0}\right) \rightarrow Z_{\mu^{\prime}}$ is a $\left(\lambda_{1}, \lambda_{2}, c_{1}, c_{2}\right)$-quasi-isometric embedding, then $f$ induces an isomorphism on fundamental groups $\pi_{1}\left(Z_{\mu}\right) \rightarrow \pi_{1}\left(Z_{\mu^{\prime}}\right)$.

Proof. We provide a proof by contradiction. Assume that for arbitrarily large values of $R$, there exists a map $f_{R}: B_{Z}(R) \rightarrow Z^{\prime}$ which is a $\left(\lambda_{1}, \lambda_{2}, c_{1}, c_{2}\right)$-quasi-isometric embedding which is not isomorphic on fundamental groups. Pick a $2 c_{1} / \lambda_{1}$-dense and $c_{1} / \lambda_{1}$-discrete subset $\Lambda$ of $Z$. Notice that if $f_{R}$ is a $\left(\lambda_{1}, \lambda_{2}, c_{1}, c_{2}\right)$-quasi-isometry, then $f_{R}$ is bi-Lipschitz on $B_{Z}(R) \cap \Lambda$. Conversely, if a map defined on $B(R) \cap \Lambda$ is bi-Lipschitz, then it can be continuously extended on $B(R)$ as a quasi-isometric embedding. Indeed, away from a ball, $Z^{\prime}$ is contractible up to scale $c_{1}$.

Set $\rho=d\left(O^{\prime}, f_{R}(O)\right)$. First, consider the case when $\rho \rightarrow \infty$. Set $\sigma=\left(\rho / 4-c_{1}\right) / \lambda_{1}$. Then $f_{R}(B(O, \sigma))$ is contained in a ball $B\left(f_{R}(O), \rho / 4\right)$ which lies in the complement of $B\left(O^{\prime}, \rho / 2\right)$

$$
f_{R}(B(O, \sigma)) \subset B\left(f_{R}(O), \rho / 4\right) \subset B\left(O^{\prime}, \rho / 2\right)^{c} .
$$

The diameter of the image of any loop in $B(O, \sigma)$ is at most $\lambda_{1} \sigma+c_{1}$. Because $\lambda_{1} \sigma+$ $c_{1}<\rho / 4$, these loops are homotopic to 0 (diameters of loops are too short relatively to $\left.B\left(O^{\prime}, \rho / 2\right)^{c}\right)$. Hence, the restriction of $f_{R}$ on $B(0, \sigma)$ is homotopic to 0 . Hence $f_{R}$ lifts to $\tilde{f}_{R}: B_{Z}(\sigma) \rightarrow \tilde{Z}^{\prime}=X_{\mu^{\prime}}$ which is homogeneous. Now up to composing $\tilde{f}_{R}$ with an isometry we can suppose that it preserves the center $\tilde{f}_{R}(O)=O^{\prime}$. By Ascoli's theorem, we can find a sequence $\left.\tilde{f}_{R_{j}}\right|_{\Lambda}$ which uniformly converges to $\tilde{f} \mid \Lambda: Z \cap \Lambda \rightarrow \tilde{Z}^{\prime}$ which is also bi-Lipschitz. We continuously extend $\tilde{f}_{\mid \Lambda}$ to $\tilde{f}: Z \rightarrow \tilde{Z}^{\prime}, \tilde{f}$ is a quasi-isometric embedding. Its extension to ideal boundaries is continuous and injective. By the theorem of invariance of domain, $\partial \tilde{f}: T^{n} \simeq \partial X_{\mu}=S^{n}$ is open, and thus a homeomorphism. This provides a contradiction if $n \geq 2$.

If $\rho=d\left(O^{\prime}, f_{R}(O)\right)$ stays bounded, we can directly use Ascoli's theorem, and get a limiting continuous quasi-isometric embedding $f$. Again, $f$ extends to the ideal boundary, $\partial f: \partial Z \rightarrow \partial Z^{\prime}$, the map $\partial f$ is continuous and injective. Because $\partial Z$ and $\partial Z^{\prime}$ have the same dimension, $\partial f$ is an open map by the theorem of invariance of domain and $\partial f$ is a homeomorphism. Hence, $\partial f$ induces an isomorphism on fundamental groups. If $R_{j}$ is sufficiently large, then $f_{R_{j}}$ is at bounded distance from $f$ and hence $f_{R_{j}}$ also induces an isomorphism $\pi_{1}\left(B_{Z}(R)\right) \rightarrow \pi_{1}\left(Z^{\prime}\right)$. This contradiction completes the proof. 
Remark 4. The proof does not provide an effective value of $R_{0}$.

\section{ACKowledgement}

The author thanks Pierre Pansu for his invaluable help through all steps of this work on the paper. This work is supported by Agence Nationale de la Recherche, grant ANR10-BLAN 0116. This work is also partially supported by RFBR, grant 14-07-00812.

\section{REFERENCES}

[1] I. Benjamini, O. Schramm, Lack of sphere packing of graphs via nonlinear potential theory. J. Topol. Anal. 5 (2013), no. 1, 1-11.

[2] I. Benjamini, O. Schramm, A. Timr, On the separation profile of infinite graphs. Groups Geom. Dyn. 6 (2012), no. 4, 639-658.

[3] P. Pansu Cohomologie $L^{p}$ des variétés à courbure négative, cas du degré 1, Rend. Semin. Mat., Torino Fasc. Spec., (1989) 95-120.

[4] N. Shanmugalingam, X. Xie, A rigidity property of some negatively curved solvable Lie groups, to appear in Comment. Math. Helv.

[5] X. Xie, Quasisymmetric maps on the boundary of a negatively curved solvable Lie group, arXiv:1001.0148 (2009), to appear in Mathematische Annalen

[6] U. Hamenstädt, Zur Theorie des Carnot-Caratheodory Metriken und ihren Anwendungen, Bonner Math. Schriften, 180 (1987)

[7] E. Heintze, On homogeneous manifolds of negative curvature. Math. Ann. 211 (1974), 2334.

[8] V. Shchur, Quasi-isometries between hyperbolic metric spaces, quantitative aspects. tel-00867709, http://tel.archives-ouvertes.fr/tel-00867709

[9] P. Papasoglu, Homogeneous trees are bilipschitz equivalent, Geometriae Dedicata, 54 (1995) 301306

[10] M. Bourdon, B. Kleiner, Some applications of $L^{p}$-cohomology to boundaries of Gromov hyperbolic spaces, arXiv:1203.1233 (2012)

[11] Ch. Croke, Some isoperimetric inequalities and eigenvalue estimates, Ann. Sci. Ec. Norm. Sup. Paris, 13 (1980) 419-535.

Université Paris-Sud, F-91405 Orsay Cedex, France

Current address: Wellcome Trust Sanger Institute, CB10 1SA Hinxton, Cambridgeshire, $\mathrm{UK}$

VLSHCHUR@GMAIL.COM

VS3@SANGER.CO.UK 